\title{
A mean-field approach to elastically coupled hair bundles
}

\author{
K. Dierkes ${ }^{1, a}$, F. Jülicher ${ }^{1}$, and B. Lindner ${ }^{2, b}$ \\ 1 Max Planck Institute for the Physics of Complex Systems, Nöthnitzer Str. 38, 01187 Dresden, Germany \\ 2 Bernstein Center for Computational Neuroscience and Physics Dep. Humboldt University Berlin, Philippstr. 13, Haus 2, \\ 10115 Berlin, Germany
}

Received 2 November 2011 and Received in final form 28 March 2012

Published online: 25 May 2012

(C) The Author(s) 2012. This article is published with open access at Springerlink.com

\begin{abstract}
We study the dynamics of oscillatory hair bundles which are coupled elastically in their deflection variable and are subject to noise. We present a stochastic description capturing the dynamics of the hair bundles' mean field. In particular, the presented derivation elucidates the origin of the previously described noise reduction by coupling. By comparison of simulations of the approximate dynamics and the full system, we verify our results. Furthermore, we demonstrate that the specific type of coupling considered implies coupling-induced changes in the dynamics beyond mere noise reduction.
\end{abstract}

\section{Introduction}

Oscillatory processes in biological systems are ubiquitous, serving a multitude of functions. Periodic variations in gene expression are key to the generation of circadian rhythms [1]. Oscillatory activity of cardiac pacemaker cells triggers contractions of the heart muscle [2]. In neuroscience, stochastic oscillations in the firing of neural populations play an essential role in diverse signal processing tasks [3-5]. Mechanical oscillations of sensory hair bundles are thought to be essential for the operation of hearing organs [6]. Theoretical analysis has helped in elucidating the diverse mechanisms responsible for the generation of oscillations as well as their functional roles [7-9].

The precision and regularity of periodic processes on cellular length and time scales is often perturbed by unavoidable fluctuations. As a consequence, the amplitude and phase of the oscillation turn into stochastic variables. In experimental data, this becomes manifest, for instance, in a considerable variability of the interbeat interval of isolated cardiac pacemaker cells [10] or in broadly peaked power spectra of hair-bundle oscillations [11].

Oscillations at large scales often rely on coupling of oscillatory or excitable elements on the cellular scale. For instance, the primary circadian clock in mammals consists of many cells, each of which is exhibiting oscillatory changes in the expression levels of certain genes [12]. Coupling, in this case, is realized by diffusion of signaling molecules. Another example is provided by the hair bundles of outer

\footnotetext{
a Current address: Centre for Genomic Regulation (CRG) and UPF, Dr. Aiguader 88, 08003 Barcelona, Spain.

b e-mail: benjamin.lindner@physik.hu-berlin.de
}

hair cells in the mammalian cochlea, which are coupled in a direct mechanical manner [13]. Bi-directional coupling has been shown to have the potential to reduce the detrimental effects of noise $[10,14,15]$, as evidenced, for example, by an increase of the regularity of the oscillatory behavior.

From a theoretical point of view, coupled noisy biological oscillators are described by high-dimensional, nonlinear, stochastic dynamics, which is in general challenging to analyze. One popular way to approach this problem is to abstract from the oscillatory process under study and to consider a more generic situation instead, namely the dynamics of coupled noisy phase oscillators (reviewed in [16]). Such ensembles of oscillators have lent themselves to numerical as well as analytical treatments. For instance, it has been shown that a synchronization transition occurs when coupling exceeds a certain threshold strength $[8,17$, 18]. In particular, theoretical results related to couplinginduced noise reduction could be derived [19]. While this approach can reveal general principles as to the effect of coupling on noisy oscillators, it cannot resolve dynamic effects due to system-specific nonlinearities and types of coupling realized in actual biological systems.

Here, we aim at an analysis of the effects of coupling on a specific biological oscillator: the sensory hair bundle. In the inner ear of vertebrates, the hair bundle serves as the transducer of mechanical stimuli into electrical signals. Hair bundles from various inner ear organs have been shown to possess the ability to actively oscillate even in the absence of any stimulation [11]. Mechanical hair bundle oscillations mark an out-of-equilibrium process that is powered by active force production of molecular motors [20]. Moreover, the hair bundle has been shown to nonlinearly 
amplify external periodic stimuli in a frequency selective way and has been attributed a key role in cochlear amplification [21,22]. However, the amplification performance of a single hair bundle is limited by intrinsic fluctuations [23]. In many inner ear organs, however, hair bundles do not operate in isolation. Within the mammalian cochlea, the hair bundles of outer hair cells are attached to the overlying tectorial membrane, a gelatinous structure which is mostly elastic but also shows signs of internal friction [24]. Estimates of its material properties suggest that it introduces a strong coupling among hair bundles [25,26]. Similarly, in the sacculus of the bullfrog, hair bundles are attached to the otolithic membrane. Its reported material properties suggest that hair bundles are strongly elastically coupled by the otolithic membrane $[27,28]$. In the basilar papilla of many lizard species, the hair bundles of the high-frequency region are coupled by tectorial structures, either in the form of a continuous membrane or in the form of a chain of so-called tectorial sallets [29]. It has been pointed out that a lizard species with incomplete tectorial structures, has an increased hearing threshold as compared to a different species that possesses those structures [29].

Recent theoretical and experimental work has shown that coupling can synchronize hair bundle movements, can increase the regularity of spontaneous oscillations, and as a consequence can enhance the sensitivity of hair bundles to external periodic stimulation $[15,30]$. As of today, however, a theoretical understanding of the origin of the observed noise reduction is still lacking.

In the following, we discuss the dynamics of systems of coupled hair bundles in the framework of a stochastic mean-field approach. To this end, we make use of an established biophysical description of single hair bundle dynamics [23,31], expressed in the form of a system of two coupled stochastic differential equations. This model was initially developed to capture the dynamics of bullfrog saccular hair bundles, an important biological model system. It has, however, also been successful in describing the behavior of hair bundles in other amphibian and mammalian systems, in particular the turtle inner ear and the rat cochlea [31].

When systems of coupled hair bundles are considered, an elastic coupling, as realized in the cochlea by the tectorial membrane or in the sacculus by the otolithic membrane, leads to a coupling term in only one of the two degrees of freedom. Motivated by the mammalian cochlea, where an overall morphological gradient exists but where nearby hair bundles have similar properties, we consider systems of coupled hair bundles with identical properties. We discuss how under such conditions an effective noise reduction comes about. Furthermore, we show that this specific type of coupling induces additional dynamic effects, such as changes in the underlying bifurcation structure. In particular, our approach gives reasonable results for all choices of system sizes, i.e. it does not presuppose an infinitely large number of coupled hair bundles.

This article is organized as follows. In sect. 2, we review the biophysical description of hair bundle motility and the type of coupling considered. Also, we briefly summarize previously reported effects of elastic coupling on the spontaneous and driven dynamics of systems of coupled hair bundles. In sect. 3, assuming strong coupling, we derive a set of stochastic differential equations approximating the dynamics of the mean field. In sect. 4, we compare simulations of the derived stochastic mean-field dynamics, of the full model of coupled hair bundles, and of a single hair bundle with reduced noise. In particular, we show that our mean-field approach is able to capture dynamic effects beyond simple noise reduction. We conclude in sect. 5 with a discussion of our results.

\section{Model of coupled hair bundles}

In this section, we recall the biophysical description of individual hair-bundle dynamics introduced in [23,31] and discuss the type of coupling among hair bundles studied in the following.

Each individual hair bundle is formed by a number of actin filled protrusions, so-called stereocilia, which emanate from the apical surface of specialized sensory cells, so-called hair cells. Stereocilia are arranged in rows of increasing height, thus defining an axis $X$ along which mechanotransduction takes place. Stereocilia confer a pivotal stiffness $K_{\mathrm{SP}}$ to the hair bundle. Small filaments, socalled tip links, connect each row of stereocilia to the next taller row of stereocilia. Upon a deflection of the hair bundle in the positive $X$-direction, the open probability $P_{o}$ of mechanically gated ion channels, attached on one or both sides of each tip-link, is increased. Tip-links introduce a gating stiffness $K_{\mathrm{GS}}$ to the hair-bundle system. Upon opening of ion channels, a transduction current can flow into the underlying hair cell. The resulting modulation of the membrane potential affects the release of neurotransmitter at the basal side of the hair cell and thus influences the generation of action potentials in the afferent neuron connected to the hair cell. The opening probability of ion channels also depends on the position of adaptation motors, denoted by $X_{a}$, which are presumably attached on the inside of stereocilia to the complex consisting of ion channel(s) and tip-link. More specifically, the open probability has a sigmoidal dependence on the relative position of $X$ with respect to $X_{a}$ and is for a simple two-state channel given by

$$
P_{o}\left(X, X_{a}\right)=1 /\left(1+A \exp \left(-\left(X-X_{a}\right) / \delta\right) .\right.
$$

Here, $A=\exp \left[\left(\Delta G+K_{\mathrm{GS}} D^{2} / 2 N_{e}\right) / k_{B} T\right]$, where $D$ is associated with channel gating and $k_{B} T$ is the thermal energy. Furthermore, $\delta=N_{e} k_{B} T / K_{\mathrm{GS}} D$, where $N_{e}$ is the total number of transduction elements within the hair bundle. The dynamic equations governing the evolution of the deflection $X$ and the motor position $X_{a}$ for an individual hair bundle read

$$
\begin{aligned}
\lambda \dot{X} & =f_{X}\left(X, X_{a}\right)+F(t)+\xi(t), \\
\lambda_{a} \dot{X}_{a} & =f_{X_{a}}\left(X, X_{a}\right)+\xi_{a}(t),
\end{aligned}
$$


Table 1. Table of standard parameters.

\begin{tabular}{lll} 
Parameter & Definition & Value \\
\hline$\lambda$ & $\begin{array}{l}\text { Friction coefficient } \\
\text { of hair bundle }\end{array}$ & $2.8 \mu \mathrm{N} \mathrm{s} \mathrm{m}^{-1}$ \\
$\lambda_{a}$ & $\begin{array}{l}\text { Friction coefficient } \\
\text { of adaptation motors }\end{array}$ & $10.0 \mu \mathrm{N} \mathrm{s} \mathrm{m}^{-1}$ \\
$K_{\mathrm{GS}}$ & $\begin{array}{l}\text { Combined stiffness } \\
\text { gating-spring stiffness }\end{array}$ & $0.75 \mathrm{mN} \mathrm{m}^{-1}$ \\
$K_{\mathrm{SP}}$ & $\begin{array}{l}\text { Combined stiffness } \\
\text { of stereociliary pivots }\end{array}$ & $0.6 \mathrm{mN} \mathrm{m}^{-1}$ \\
$D$ & $\begin{array}{l}\text { Gating-swing } \\
\text { on channel opening }\end{array}$ & $62.14 \mathrm{~nm}$ \\
$N_{e}$ & $\begin{array}{l}\text { Number of transduction } \\
\text { elements }\end{array}$ & 50 \\
$T$ & Ambient temperature & $300 \mathrm{~K}$ \\
$T_{a}$ & Effective temperature & $1.5 \mathrm{~T}$ \\
$\Delta G$ & Intrinsic energy change & $10 k_{B} T$ \\
$F_{\max }$ & on channel opening \\
$S$ & Maximal motor force & $49.23 \mathrm{pN}$ \\
\hline
\end{tabular}

where $\lambda$ and $\lambda_{a}$ are friction coefficients and the right-hand sides are given by

$$
\begin{aligned}
f_{X}\left(X, X_{a}\right) & =-K_{\mathrm{GS}}\left(X-X_{a}-D P_{o}\right)-K_{\mathrm{SP}} X \\
f_{X_{a}}\left(X, X_{a}\right) & =K_{\mathrm{GS}}\left(X-X_{a}-D P_{o}\right)+F_{\max }\left(S P_{o}-1\right) .
\end{aligned}
$$

Note that the force exerted by the gating springs depends on the open probability of the ion channels. Effectively, this gating compliance, which is related to a change in tip-link tension upon opening and closing of the ion channels, renders hair-bundle stiffness a nonlinear function of displacement [32]. Molecular motors can produce a maximal force $F_{\max }$. However, upon opening of ion channels, inflowing calcium inhibits the motor. The strength of this negative feedback is set by the dimensionless parameter $S$. The hair bundle is subject to fluctuations, stemming from the Brownian motion of the tip of the hair bundle in the surrounding bath, the stochastic opening and closing of ion channels, and a stochastic component due to the force production of molecular motors. In order to account for these fluctuations, eq. (2) contains zero mean Gaussian white noise terms $\xi(t)$ and $\xi_{a}(t)$, respectively, with correlation functions $\langle\xi(t) \xi(t+\tau)\rangle=\varepsilon 2 \lambda k_{B} T \delta(\tau)$ and $\left\langle\xi_{a}(t) \xi_{a}(t+\tau)\right\rangle=\varepsilon 2 \lambda_{a} k_{B} T_{a} \delta(\tau)$. Note the effective temperature $T_{a} \approx 1.5 T$, appearing in the correlation function of $\xi_{a}(t)$, is related to the presence of out-of-equilibrium fluctuations in the motor force [23]. Furthermore, for later use we have introduced a dimensionless noise reduction factor $\varepsilon$. An external mechanical force acting on the tip of the hair bundle is included by the term $F(t)$.

When choosing parameters according to table 1 and considering $S$ and $F_{\max }$ as free parameters, the hairbundle model in the undriven deterministic case, i.e. for $F(t)=0$ and $\varepsilon=0$, was shown to exhibit a parameter

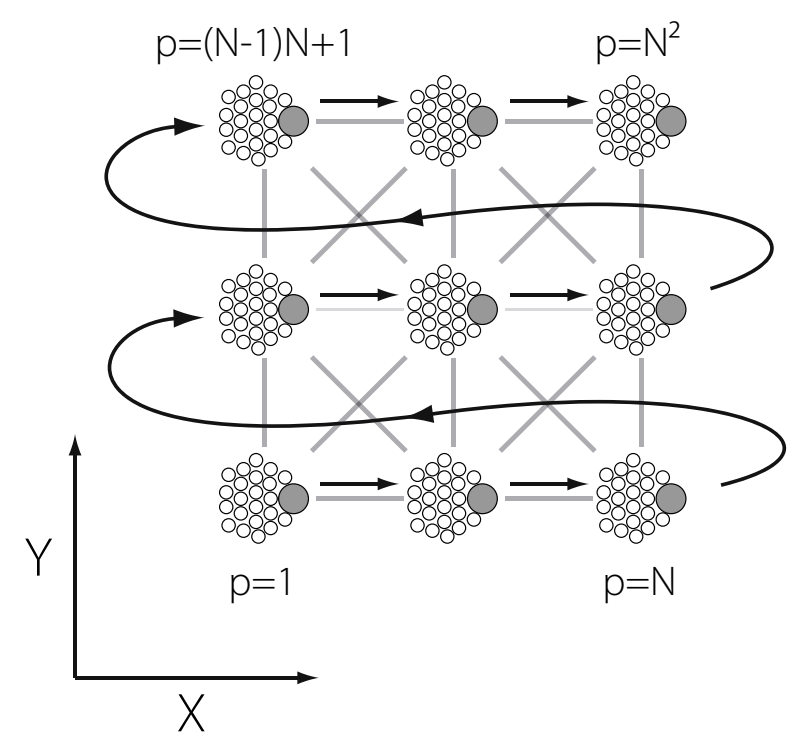

Fig. 1. Schematic of a system of $N \times N$ coupled hair bundles. Starting from the left lower corner, each row of hair bundles is labeled with a running index, $p$. Mechanical oscillations of hair bundles occur along the $X$-axis. Movements along the $Y$-axis are not considered. Grey lines indicate elastic springs mediating coupling among hair bundles.

region bounded by Hopf bifurcation lines, in which the system performs limit-cycle oscillations (cf. sect. 4). When placed in or near this region, in the presence of noise the system thus shows noisy limit cycle oscillations. For a choice of parameters corresponding to table 1, these oscillations quantitatively resemble hair bundle oscillations as observed for isolated hair bundles from the sacculus of the bullfrog [23]. Furthermore, also the response of this hair bundle system to external driving matches quantitatively the behavior observed experimentally [23]. We have chosen a set of parameters studied previously [23,30,33] (see table 1), which we will refer to as standard parameters. Unless indicated otherwise, simulations were performed using these standard parameters.

We now turn to systems of coupled hair bundles. We will assume the same type and topology of coupling as introduced in [30]. Hair bundles with identical parameters are arranged on a square lattice with lattice spacing $d$. We restrict our attention to quadratic systems of $N \times N$ hair bundles with system size $N^{2}$. For notational purposes, it is useful to label hair bundles in a different manner as compared to [30], namely by a single index, $p=1 \ldots N^{2}$, as shown in fig. 1 . Thus, for each hair bundle there exists a unique pair $(i, j)$ with $1 \leq i \leq N$ and $1 \leq j \leq N$, such that $p=(j-1) N+i$. The dynamic state of each hair bundle is now given in terms of its deflection $X_{p}$ and motor position $X_{a, p}$. Note, we are ignoring deflections along the $Y$-axis. Hair bundles are subject to pairwise independent noise terms $\xi_{p}(t)$ and $\xi_{a, p}(t)$. In the coupled system, we exclusively consider the case $\varepsilon=1$.

The response of the tectorial membrane with respect to a shearing stimulus exhibits both elastic and viscous 
components [24]. Measurements of its shear impedance, however, suggest that deviations from a purely elastic material are negligible for frequencies below at least $2 \mathrm{kHz}$ [24]. Therefore, in the following we neglect viscous contributions in the coupling and describe coupling by elastic springs of stiffness $K$, which connect nearest and next-nearest neighbors (cf. fig. 1). More specifically, the hair bundle with label $p=(j-1) N+i$ is coupled to all hair bundles with labels $q=(j-1+l) N+(i+k)$, where $k, l=-1,0,1$ and $1 \leq(j+l) \leq N$ as well as $1 \leq(i+k) \leq N$. The corresponding spring force, $F_{p, q}$, is then given by

$$
F_{p, q}=K\left(1-\frac{L_{0}}{\sqrt{\left(\Delta X_{p, q}+k d\right)^{2}+(l d)^{2}}}\right)\left(\Delta X_{p, q}+k d\right),
$$

where

$$
\Delta X_{p, q}=X_{q}-X_{p}
$$

and

$$
L_{0}=\sqrt{\left(k^{2}+l^{2}\right) d^{2}}
$$

is the spring's resting length.

The full dynamics of the hair bundle with label $p$ in a system of coupled hair bundles is given by

$$
\begin{aligned}
\lambda \dot{X}_{p} & =f_{X}\left(X_{p}, X_{a, p}\right)+\sum_{q}{ }^{\prime} F_{p, q}+F(t)+\xi_{p}(t), \\
\lambda_{a} \dot{X}_{a, p} & =f_{X_{a}}\left(X_{p}, X_{a, p}\right)+\xi_{a, p}(t),
\end{aligned}
$$

where the prime in eq. (4) indicates that summation is restricted to nearest and next-nearest neighbors [30]. We will consider open boundary conditions only.

In the remainder of this section, we will shortly report on some of the previously observed effects of elastic coupling on hair-bundle dynamics [30,15]. Given a $N \times N$ system of hair bundles, in the absence of coupling, i.e. for $K=0 \mathrm{pN} / \mathrm{nm}$, hair bundle oscillations are pairwise uncorrelated (see fig. 2a, upper panel). Upon a sufficient increase of the coupling strength, oscillations progressively synchronize (see fig. $2 \mathrm{a}$, middle and lower panel). Along with synchronization, oscillations become more regular. This is, e.g., evidenced by the power spectral densities $S(f)$ shown in fig. 2b. The latter is defined by $S(f)=\lim _{T \rightarrow \infty}\left\langle|\tilde{X}|^{2}\right\rangle / T$, where $\tilde{X}(f)=\int_{0}^{T} \mathrm{~d} t X(t) e^{2 \pi i f t}$ is the Fourier transform of a hair bundle's deflection and $\langle\ldots\rangle$ denotes the average over a stationary ensemble. For various $N$, we show the power spectral density of the central hair bundle, i.e. the hair bundle located at position $p=\left(N^{2}+1\right) / 2$ in the case of odd, and $p=N^{2} / 2-N / 2$ in the case of even $N$. Note that, all systems have been poised in the tightly synchronized regime $(K=100 \mathrm{pN} / \mathrm{nm})$. For each system size, a well-defined peak is present in the power spectrum, defining a characteristic frequency $f_{0}$ of the oscillation. Note that, with increasing $N$, the frequency of oscillation undergoes a slight change. More importantly, with increasing $N$, a pronounced sharpening of the spectral peak can be discerned. One way to quantify the regularity of noisy oscillations is by means of the (a)

(b)

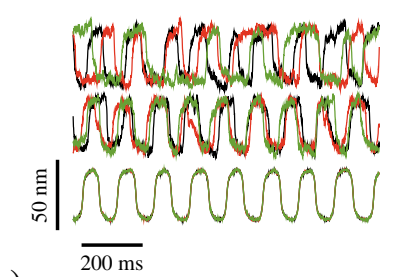

(c)
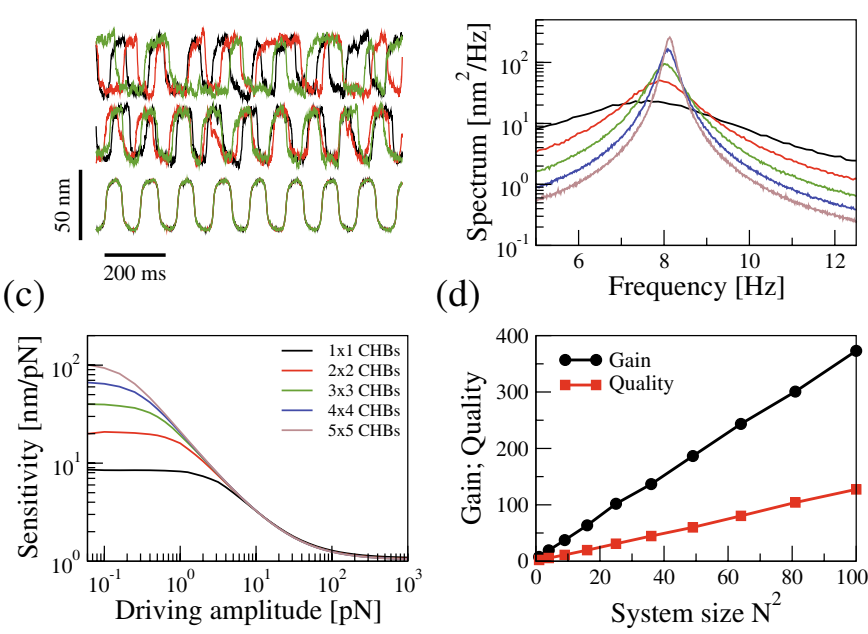

(d)

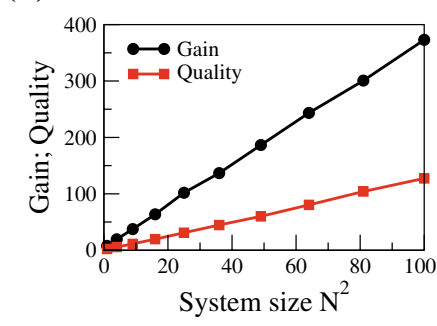

Fig. 2. (Colour on-line) Review of coupling-induced effects on hair-bundle dynamics. In (a), for three coupling strengths (upper panel, $K=0 \mathrm{pN} / \mathrm{nm}$; middle panel, $K=0.3 \mathrm{pN} / \mathrm{nm}$; lower panel, $K=100 \mathrm{pN} / \mathrm{nm})$ trajectories of three hair bundles in a $10 \times 10$ systems (black, $p=1$; red, $p=50$; green, $p=100$ ) are shown. With increasing coupling strength, hair bundle oscillations exhibit progressive synchronization. In (b), power spectra of the central hair bundle in systems of different sizes are displayed (legend in (c)). With increasing system size, a pronounced sharpening of the spectral peak at the characteristic frequency $f_{0}$ is found. For the same system sizes as in (b), the sensitivity of the central hair bundle for a sinusoidal driving at the respective characteristic frequency is shown in (c) as a function of driving amplitude. In (d), the quality factor and gain of the central hair bundle are shown as a function of system size, exhibiting a linear increase. Coupling strengths in (b), (c), and (d) are $K=100 \mathrm{pN} / \mathrm{nm}$. All simulations of coupled hair bundles here and throughout the rest of the paper were performed with a time step $\mathrm{d} t=0.001 \mathrm{~ms}$. System parameters, when not indicated otherwise, were chosen as standard parameters (see table 1).

quality factor, $Q$, which is defined as

$$
Q=f_{0} / \Delta f
$$

where $\Delta f$ is the width of the power spectrum at half its height. As shown in fig. $2 \mathrm{~d}$, the quality of spontaneous oscillations increases almost linearly with $N^{2}$.

Coupling also enhances the sensitivity of a hair bundle to weak periodic driving. In particular, we will consider a time-dependent driving of the form

$$
F(t)=F_{0} \cos (2 \pi \nu t),
$$

where we will refer to $F_{0}$ as the driving amplitude and to $\nu$ as the driving frequency. The sensitivity $\left|\chi_{\nu}\right|\left(F_{0}\right)$ of a hair bundle is defined as

$$
\left|\chi_{\nu}\right|\left(F_{0}\right)=|\langle\tilde{X}(\nu) / \tilde{F}(\nu)\rangle|
$$

where $\tilde{F}(\nu)=\int_{0}^{T} \mathrm{~d} t F(t) e^{2 \pi i \nu t}$ is the Fourier transform of the forcing. In the following, we will be interested in the 
sensitivity at the characteristic frequency of the spontaneous oscillations, i.e. the case $\nu=f_{0}$. In fig. 2c, we show the sensitivity as a function of driving amplitude for various system sizes. Note that, while coupling has almost no effect on sensitivity in the limit of strong driving amplitudes, sensitivity is increased for weak driving amplitudes. Plotting the so-called amplification gain, i.e. the ratio of sensitivity in the limit of weak driving to sensitivity in the limit of strong driving, a linear increase as a function of system size is found (see fig. $2 \mathrm{~d}$ ).

Both the linear increase of quality factor and amplification gain as a function of system size have been interpreted as being due to an effective coupling-induced noise reduction. A hair bundle in a strongly coupled system of hair bundles has been argued to obey the same dynamic equations as a single hair bundle [33], albeit with a noise strength that is reduced in proportion with system size, corresponding to a choice $\varepsilon=1 / N^{2}$. In the following, we show in what sense coupling indeed leads to an effective noise reduction. Furthermore, we show that, since coupling is only present in one degree of freedom, i.e. motor variables are not directly coupled, besides an effective noise reduction coupling also leads to qualitative changes in hair-bundle dynamics.

\section{Stochastic mean-field dynamics}

\subsection{Approximating mean-field dynamics in the limit of strong coupling}

Assuming that the coupling constant $K$ is chosen sufficiently large, hair bundles do oscillate in near synchrony (see fig. 2a). Concerted hair bundle movements give rise to a non-zero average deflection, i.e. it creates a non-zero mean field. The latter is given in terms of the average deflection $\bar{X}$ and the average motor position $\bar{X}_{a}$ of all hair bundles in the system, i.e.

$$
\bar{X}=\frac{1}{N^{2}} \sum_{p=1}^{N^{2}} X_{p} \quad \text { and } \quad \bar{X}_{a}=\frac{1}{N^{2}} \sum_{p=1}^{N^{2}} X_{a, p} .
$$

We will refer to these new dynamic variables as meanfield variables or simply as the mean field. The deviations $\delta_{p}$ of the individual deflections and $\delta_{a, p}$ of the individual motor positions from the respective mean-field variables are given by

$$
\delta_{p}=X_{p}-\bar{X} \quad \text { and } \quad \delta_{a, p}=X_{a, p}-\bar{X}_{a} .
$$

Note that the above definitions imply that

$$
\sum_{p=1}^{N^{2}} \delta_{p}=0 \quad \text { and } \quad \sum_{p=1}^{N^{2}} \delta_{a, p}=0 .
$$

In the tightly synchronized regime, the deviations $\delta_{p}$ are generally small (note the sharply peaked densities in fig. 3c, middle panel), implying that the deflection $X_{p}(t)$ of a single hair bundle within the network is close to the (a)

(b)
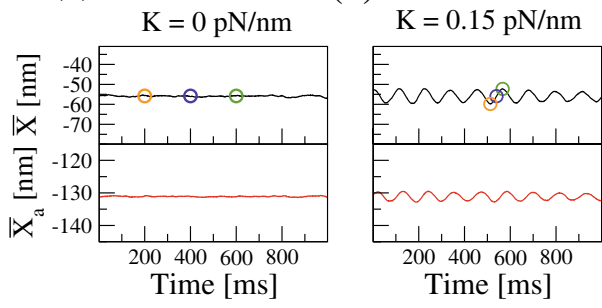

(c)
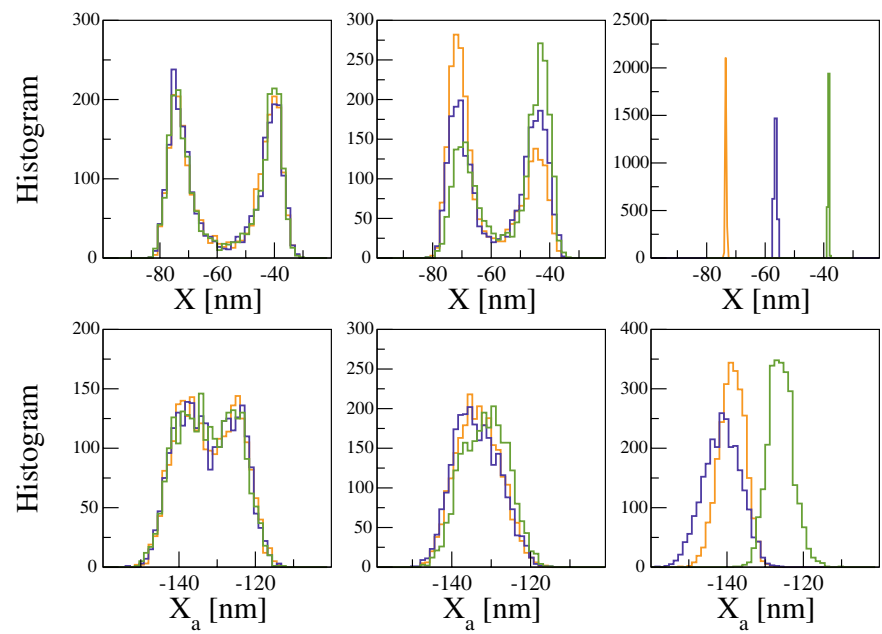

Fig. 3. (Colour on-line) Coupling-induced oscillations of the mean field. For a $50 \times 50$ system of coupled hair bundles, in the upper panels, we show trajectories of the respective meanfield variables $\bar{X}$ (black) and $\bar{X}_{a}$ (red). For vanishing coupling strength (see (a)), the mean field shows mere finite-size fluctuations about a mean value. With increasing coupling strength (see (b) and (c)) synchronization of hair bundles is reflected in an oscillatory behavior of the mean field, reminiscent of single hair bundle dynamics. Below, histograms of the individual hair bundle deflections, $X_{p}$ (middle panels), and motor positions, $X_{a, p}$ (lower panels), are shown for three points in time, respectively. Time points are indicated in the upper panel as circles of corresponding color. In the absence of coupling, histograms fluctuate about a stationary distribution. For weak coupling, modulations of the respective bimodal histograms reflect the emergence of a time-dependent mean field. In the limit of strong coupling, hair bundle deflections are tightly synchronized and the corresponding histograms are unimodal and sharply peaked. As motors are not coupled directly, histograms retain a finite width. As hair bundle deflections, however, are virtually identical at each point in time, motor variables are driven towards a preferred position on the limit cycle, evidenced by a non-constant time-dependent population average. All simulations were performed with standard parameters (see table 1).

mean field $\bar{X}(t)$ at any time. Consequently, in this regime, knowing the deflection dynamics of the mean field is equivalent to knowing the dynamics of each hair bundle in the system. Note, however, that, as motor variables are not coupled directly, also in the limit of infinite coupling, the deviations $\delta_{a, p}$ will remain finite (see fig. 3c, lower panel). In the following, we derive approximate dynamic equations for the mean-field variables. It will turn out that 
motor deviations lead to qualitative changes in mean-field dynamics as compared to a single isolated hair bundle with reduced noise.

To this end, as a first step we linearize the coupling term describing elastic hair-bundle interactions in eq. (4). Note that typical deflection amplitudes of hair bundles are on the order of several tens of nanometers. The lattice constant, however, which also sets the rest length of the springs mediating the elastic coupling, was chosen to be $d=50 \mu \mathrm{m}$. It is therefore reasonable to expand coupling forces up to linear order in relative deflections $\Delta X_{p, q}=$ $X_{q}-X_{p}$ of interacting hair bundles, leading to

$$
F_{p, q} \approx\left\{\begin{array}{cl}
K \Delta X_{p, q} & \text { for springs in } X \text {-direction } \\
\frac{1}{2} K \Delta X_{p, q} & \text { for diagonal springs } \\
0 & \text { for springs in } Y \text {-direction. }
\end{array}\right.
$$

In particular, note that springs in $Y$-direction in this approximation do not add to the elastic interactions induced by coupling and that these forces are independent of the lattice spacing $d$. Using these linearized coupling forces, the deflection dynamics for a hair bundle in the coupled system reads

$$
\begin{aligned}
\lambda \dot{X}_{p}= & f_{X}\left(X_{p}, X_{a, p}\right)+F_{\text {ext }}(t)+\xi_{p}(t) \\
& +K \sum_{m, n=-1}^{1},\left(1-\frac{|n|}{2}\right)|m| \Delta X_{p, p+n N+m} .
\end{aligned}
$$

In order to further simplify the notation, we introduce the coupling matrix $\mathbf{C}=\left(c_{p, q}\right)$. It is uniquely defined by the requirement that for all $p$ with $1 \leq p \leq N^{2}$

$$
\sum_{m, n=-1}^{1}\left(1-\frac{|n|}{2}\right)|m| \Delta X_{p, p+n N+m}=\sum_{q=1}^{N^{2}} c_{p, q} X_{q} .
$$

Because $\Delta X_{p, q}=X_{q}-X_{p}$, the matrix elements $c_{p, q}$ can be obtained from eq. (8) by equating coefficients. In particular, note that $\mathbf{C}$ is symmetric and that

$$
\sum_{q=1}^{N^{2}} c_{p, q}=0
$$

for all $p$ with $1 \leq p \leq N^{2}$. The full dynamics for a hair bundle in the coupled system can thus be written as

$$
\lambda \dot{X}_{p}=f_{X}\left(X_{p}, X_{a, p}\right)+K \sum_{q=1}^{N^{2}} c_{p, q} X_{q}+F_{\text {ext }}(t)+\xi_{p}(t),
$$

$\lambda_{a} \dot{X}_{a, p}=f_{X_{a}}\left(X_{p}, X_{a, p}\right)+\xi_{a, p}(t)$.

In order to obtain equations for the mean field, we express the right hand sides of these equations in terms of the mean-field variables $\bar{X}, \bar{X}_{a}$ and the deviations $\delta_{p}, \delta_{a, p}$. To this end, we expand the nonlinear functions $f_{X}, f_{X_{a}}$ around the mean field and simplify the coupling term using eq. (9)

$$
\begin{aligned}
\lambda \dot{X}_{p}= & \sum_{i, j=0}^{\infty} \frac{1}{i ! j !}\left[\partial_{i, j} f_{X}\left(\bar{X}, \bar{X}_{a}\right)\right] \delta_{p}^{i} \delta_{a, p}^{j} \\
& +K \sum_{q=1}^{N^{2}} c_{p, q} \delta_{q}+F_{\text {ext }}(t)+\xi_{p}(t), \\
\lambda_{a} \dot{X}_{a, p}= & \sum_{i, j=0}^{\infty} \frac{1}{i ! j !}\left[\partial_{i, j} f_{X_{a}}\left(\bar{X}, \bar{X}_{a}\right)\right] \delta_{p}^{i} \delta_{a, p}^{j}+\xi_{a, p}(t),
\end{aligned}
$$

where we define

$$
\partial_{i, j} f_{X}\left(\bar{X}, \bar{X}_{a}\right)=\left.\frac{\partial^{i+j} f_{X}\left(X, X_{a}\right)}{\partial X^{i} \partial X_{a}^{j}}\right|_{X=\bar{X}, X_{a}=\bar{X}_{a}} .
$$

We can therefore derive the following dynamic equations for the mean field:

$$
\begin{aligned}
\lambda \dot{\bar{X}}= & \frac{1}{N^{2}} \sum_{p=1}^{N^{2}} \lambda \dot{X}_{p} \\
= & f_{X}\left(\bar{X}, \bar{X}_{a}\right)+F_{\text {ext }}(t)+\frac{1}{N^{2}} \sum_{p=1}^{N^{2}} \xi_{p}(t) \\
& +\frac{1}{N^{2}} \sum_{p=1}^{N^{2}} \sum_{\substack{i, j=0 \\
i+j>1}}^{\infty} \frac{1}{i ! j !}\left[\partial_{i, j} f_{X}\left(\bar{X}, \bar{X}_{a}\right)\right] \delta_{p}^{i} \delta_{a, p}^{j}, \\
\lambda_{a} \dot{\bar{X}}_{a}= & \frac{1}{N^{2}} \sum_{p=1}^{N^{2}} \lambda_{a} \dot{X}_{a, p} \\
= & f_{X_{a}}\left(\bar{X}, \bar{X}_{a}\right)+\frac{1}{N^{2}} \sum_{p=1}^{N^{2}} \xi_{a, p}(t) \\
& +\frac{1}{N^{2}} \sum_{p=1}^{N^{2}} \sum_{\substack{i, j=0 \\
i+j>1}}^{\infty} \frac{1}{i ! j !}\left[\partial_{i, j} f_{X_{a}}\left(\bar{X}, \bar{X}_{a}\right)\right] \delta_{p}^{i} \delta_{a, p}^{j} .
\end{aligned}
$$

Note that the coupling term and the linear terms in the Taylor expansion drop out because of eq. (9) and eq. (7), respectively. For the noise term in the first equation one finds

$$
\begin{aligned}
\left\langle\frac{1}{N^{2}} \sum_{q=1}^{N^{2}} \xi_{q}(t) \frac{1}{N^{2}} \sum_{p=1}^{N^{2}} \xi_{p}\left(t^{\prime}\right)\right\rangle & =\frac{1}{N^{4}} \sum_{p=1}^{N^{2}}\left\langle\xi_{p}(t) \xi_{p}\left(t^{\prime}\right)\right\rangle \\
& =\frac{2 \lambda k_{B} T}{N^{2}} \delta\left(t-t^{\prime}\right),
\end{aligned}
$$

as the white noise terms $\xi_{p}(t)$ are pairwise uncorrelated and $\varepsilon=1$ in the coupled case. A similar argument holds true for the sum of noise sources appearing in the equation for $\bar{X}_{a}$. As the sum of Gaussian variables is again Gaussian, we can therefore substitute the sums of noise sources appearing in eq. (10) by two effective white noise terms $\sqrt{1 / N^{2}} \bar{\xi}(t)$ and $\sqrt{1 / N^{2}} \bar{\xi}_{a}(t)$ with respective autocorrelation functions $\left\langle\bar{\xi}(t) \bar{\xi}\left(t^{\prime}\right)\right\rangle=2 \lambda k_{B} T \delta\left(t-t^{\prime}\right)$ and $\left\langle\bar{\xi}_{a}(t) \bar{\xi}_{a}\left(t^{\prime}\right)\right\rangle=2 \lambda_{a} k_{B} T_{a} \delta\left(t-t^{\prime}\right)$. 
In order to simplify eq. (10), we note that deviations $\delta_{p}$ can be made arbitrarily small, if $K$ is chosen sufficiently large. To illustrate this point, we consider the dynamics of the $\delta_{p}$ (all terms divided by the coupling strength):

$$
\begin{aligned}
\frac{\lambda}{K} \dot{\delta}_{p}= & \frac{1}{K}\left[\lambda \dot{X}_{p}-\lambda \dot{\bar{X}}\right] \\
= & \frac{1}{K} f_{X}\left(\bar{X}+\delta_{p}, \bar{X}+\delta_{a, p}\right) \\
& -\frac{1}{K N^{2}} \sum_{q=1}^{N^{2}} f_{X}\left(\bar{X}+\delta_{q}, \bar{X}_{a}+\delta_{a, q}\right) \\
& +\frac{1}{K}\left(\xi_{p}(t)-\frac{1}{N^{2}} \sum_{q=1}^{N^{2}} \xi_{q}(t)\right)+\sum_{q=1}^{N^{2}} c_{p, q} \delta_{q} .
\end{aligned}
$$

Because $f_{X}$ and $f_{X_{a}}$ are continuous and for large arguments approach linear functions, their contribution becomes negligible when $K$ becomes large. The remaining terms correspond to a multidimensional OrnsteinUhlenbeck process, for which one can show that the variance and the correlation time scale inversely with $K$. Hence, in the limit of infinite $K$, the contribution of $\delta_{p}$ in eq. (10) can and will be neglected.

When considering the equivalent dynamic equation for $\delta_{a, p}$, i.e.

$$
\begin{aligned}
\lambda_{a} \dot{\delta}_{a, p}= & \lambda_{a} \dot{X}_{a, p}-\lambda_{a} \dot{\bar{X}}_{a} \\
= & f_{X_{a}}\left(\bar{X}+\delta_{p}, \bar{X}_{a}+\delta_{a, p}\right) \\
& -\frac{1}{N^{2}} \sum_{q=1}^{N^{2}} f_{X_{a}}\left(\bar{X}+\delta_{q}, \bar{X}_{a}+\delta_{a, q}\right) \\
& +\left(\xi_{a, p}(t)-\frac{1}{N^{2}} \sum_{q=1}^{N^{2}} \xi_{a, q}(t)\right),
\end{aligned}
$$

no such argument applies, because the coupling is only in the deflection variables and, consequently, the large parameter $K$ does not appear here. This becomes manifest in the histograms in fig. 3, lower panels, which retain a finite variance even in the limit of large values of $K$. The variance of the $\delta_{a, p}$ in this limit is set by the effective temperature $T_{a}$, which determines the noise strength acting on the motor variables. In particular, for vanishing $T_{a}$, we expect that also the $\delta_{a, p}$ become arbitrarily small.

Motivated by this, we will concentrate on the first nonzero term in the expansions appearing in eq. (10), i.e. terms of the order $\delta_{a, p}^{2}$. The equations for the mean field accordingly reduce to

$$
\begin{aligned}
\lambda \dot{\bar{X}}= & f_{X}\left(\bar{X}, \bar{X}_{a}\right)+F_{\text {ext }}(t)+\sqrt{\frac{1}{N^{2}}} \bar{\xi}(t) \\
& +\frac{1}{2}\left[\partial_{0,2} f_{X}\left(\bar{X}, \bar{X}_{a}\right)\right] \bar{Z}, \\
\lambda_{a} \dot{\bar{X}}_{a}= & f_{X_{a}}\left(\bar{X}, \bar{X}_{a}\right)+\sqrt{\frac{1}{N^{2}}} \bar{\xi}_{a}(t) \\
& +\frac{1}{2}\left[\partial_{0,2} f_{X_{a}}\left(\bar{X}, \bar{X}_{a}\right)\right] \bar{Z},
\end{aligned}
$$

where we have introduced the new variable $\bar{Z}$ as

$$
\bar{Z}=\frac{1}{N^{2}} \sum_{p=1}^{N^{2}} \delta_{a, p}^{2}
$$

to denote the population variance of the deviations $\delta_{a, p}$. In the next step, we will derive the dynamic equations governing the time evolution of $\bar{Z}$. To this end, note that to linear order in $\delta_{a, p}$, we have

$$
\lambda_{a} \dot{\delta}_{a, p}=-a(t) \delta_{a, p}+\xi_{a, p}(t)-\frac{1}{N^{2}} \sum_{q=1}^{N^{2}} \xi_{a, q}(t) .
$$

Here, we have introduced

$$
a(t)=-\partial_{0,1} f_{X_{a}}\left(\bar{X}, \bar{X}_{a}\right) .
$$

A formal solution of eq. (15) is given by

$$
\begin{aligned}
& \delta_{a, p}(t)= \\
& \frac{1}{\lambda_{a}} e^{\Phi(t)} \int_{-\infty}^{t} \mathrm{~d} s\left(\xi_{a, p}(s)-\frac{1}{N^{2}} \sum_{q=1}^{N^{2}} \xi_{a, q}(s)\right) e^{-\Phi(s)},
\end{aligned}
$$

where

$$
\Phi(t)=-\int_{0}^{t} \mathrm{~d} s \frac{a(s)}{\lambda_{a}} .
$$

Note that, for positive $t$, eq. (16) describes the stationary properties of $\delta_{a, p}$, which are independent of initial conditions at $t=-\infty$.

By definiton of the variable $\bar{Z}$ we find

$$
\begin{aligned}
\dot{\bar{Z}} & =\frac{1}{N^{2}} \sum_{p=1}^{N^{2}} 2 \delta_{a, p} \dot{\delta}_{a, p} \\
& =\frac{2}{\lambda_{a}}\left(-a(t) \bar{Z}+\frac{1}{N^{2}} \sum_{p=1}^{N^{2}} \delta_{a, p} \xi_{a, p}(t)\right)
\end{aligned}
$$

where we have made use of eq. (7). This means that

$$
\frac{\lambda_{a}}{2} \dot{\bar{Z}}=-a(t) \bar{Z}+\eta(t)
$$

where we have introduced the noise term $\eta$ defined as

$$
\eta(t)=\frac{1}{N^{2}} \sum_{p=1}^{N^{2}} \delta_{a, p} \xi_{a, p}(t)
$$

In order to be able to utilize eq. (19) as a dynamic equation for $\bar{Z}$ without having to keep track of the $\delta_{a, p}$ explicitly, one needs to determine the correlation function of the noise term $\eta(t)$. In appendix B, we show that the mean value of $\eta$ is given by

$$
\langle\eta(t)\rangle \approx\left(1-\frac{1}{N^{2}}\right) k_{B} T_{a}
$$


and the correlation function of $\eta$ is given by

$$
\begin{aligned}
\langle\eta(t) \eta(t+\tau)\rangle \approx & \left(2 k_{B} T_{a}\right)^{2}\left(\frac{1}{N^{2}}-\frac{1}{N^{4}}\right) D_{\bar{Z}} \delta(\tau) \\
& +\left(1-\frac{1}{N^{2}}\right)^{2}\left(k_{B} T_{a}\right)^{2}
\end{aligned}
$$

where we have introduced the time-dependent noise strength $D_{\bar{Z}}$. The latter is given as the solution of the deterministic differential equation

$$
\dot{D}_{\bar{Z}}=-\frac{2}{\lambda_{a}} a(t) D_{\bar{Z}}+1
$$

Summing up, we have found an effective description of the mean-field dynamics in terms of four differential equations, three of which contain noise terms. These equations read:

$$
\begin{aligned}
\lambda \dot{\bar{X}}= & f_{X}\left(\bar{X}, \bar{X}_{a}\right)+F_{\text {ext }}(t)+\sqrt{\frac{1}{N^{2}}} \bar{\xi}(t) \\
& +\frac{1}{2}\left[\partial_{0,2} f_{X}\left(\bar{X}, \bar{X}_{a}\right)\right] \bar{Z} \\
\lambda_{a} \dot{\bar{X}}_{a}= & f_{X_{a}}\left(\bar{X}, \bar{X}_{a}\right)+\sqrt{\frac{1}{N^{2}} \bar{\xi}_{a}(t)} \\
& +\frac{1}{2}\left[\partial_{0,2} f_{X_{a}}\left(\bar{X}, \bar{X}_{a}\right)\right] \bar{Z} \\
\frac{\lambda_{a}}{2} \dot{\bar{Z}}= & {\left[\partial_{0,1} f_{X_{a}}\left(\bar{X}, \bar{X}_{a}\right)\right] \bar{Z}+\left(1-\frac{1}{N^{2}}\right) k_{B} T_{a} } \\
& +2 k_{B} T_{a} \sqrt{\left(\frac{1}{N^{2}}-\frac{1}{N^{4}}\right) D_{\bar{Z}} \hat{\eta}(t)} \\
\dot{D}_{\bar{Z}}= & \frac{2}{\lambda_{a}}\left[\partial_{0,1} f_{X_{a}}\left(\bar{X}, \bar{X}_{a}\right)\right] D_{\bar{Z}}+1,
\end{aligned}
$$

where $\hat{\eta}(t)$ is delta-correlated white noise with

$$
\langle\hat{\eta}(t) \hat{\eta}(t+\tau)\rangle=\delta(\tau)
$$

The noise terms $\bar{\xi}(t), \bar{\xi}_{a}(t)$, and $\hat{\eta}(t)$ are pairwise uncorrelated as shown in appendix A.

Note that the dynamics for $\bar{Z}(t)$ in eq. (22) can become unstable as we have dropped stabilizing nonlinearities from the Taylor expansion in eq. (10). Indeed, in simulations of eq. (22) for small $N$ we observe that the population variance can in rare cases go to unphysical ranges, i.e. it can attain negative or very large positive values. Therefore, we impose reflecting boundary conditions at $\bar{Z}=0$ and $\bar{Z}=\bar{Z}_{\max }$, which mimic the effect of saturating nonlinearities. In our simulations, we have chosen $\bar{Z}_{\max }=70 \mathrm{~nm}^{2}$, a value that is well above the variance of the motor positions in the uncoupled case.

For $N=1$, the dynamics given in eq. (22) reduces to the original description of hair bundle dynamics in eq. (1). For $N>1$, we find that the strengths of noise terms acting on $\bar{X}$ and $\bar{X}_{a}$, respectively, are reduced by $N^{2}$, i.e. inversely proportional to system size. This effect corresponds to the observed noise reduction in systems of coupled hair bundles.
However, our analysis reveals that coupling also leads to additional terms in the dynamics of the mean field, which are proportional to the variance $\bar{Z}$ of motor deviations $\delta_{a, p}$. We emphasize that this additional degree of freedom, $\bar{Z}$, arises in the mean-field description because only the deflection variables are elastically coupled. Conversely, if both hair bundle deflection and motor position were coupled between hair bundles, the resulting meanfield dynamics would correspond to the dynamics of a single hair bundle with reduced noise, i.e. without the additional degree of freedom $\bar{Z}$.

For a pure coupling in the deflection variable, the additional variable $\bar{Z}$ does not scale with the inverse system size and, in particular, remains finite for $N \rightarrow \infty$. Thus, while in the limit of infinite $N$, all noise terms in eq. (22) vanish, the individual motor variables are still distributed around a mean-field value. In sect. 4 , we will show that in the limit of infinite $N$ qualitative differences of the dynamics of the mean field as compared to a single deterministic hair bundle emerge. These quasi-deterministic changes to hair bundle dynamics due to coupling are shown to also have an effect on the statistics of hair bundle dynamics in the case of finite $N$.

\subsection{When is this a good approximation?}

In this subsection, we will discuss under what conditions the stochastic mean-field dynamics in eq. (22) is expected to give a good approximation to the mean field of a system of coupled hair bundles. Note that an approximation was necessary since for finite $T_{a}$ the distribution of $\delta_{a, p}$ retains a finite width, even for arbitrarily large coupling strengths $K$. In the following, we give an estimate up to what effective temperature reasonable results are to be expected. To this end, note first that because

$$
\frac{\partial P_{o}}{\partial X_{a}}=-P_{o}\left(1-P_{o}\right) / \delta
$$

one has

$$
\begin{aligned}
\frac{\partial^{n} f_{X_{a}}}{\partial X_{a}^{n}} & =\left(F_{\max } S-K_{\mathrm{GS}} D\right) \frac{\partial^{n} P_{o}}{\partial X_{a}^{n}} \\
& =\frac{(-1)^{n}}{\delta^{n}}\left(F_{\max } S-K_{\mathrm{GS}} D\right) F_{n}\left(P_{o}\right)
\end{aligned}
$$

and

$$
\begin{aligned}
\frac{\partial^{n} f_{X}}{\partial X_{a}^{n}} & =K_{\mathrm{GS}} D \frac{\partial^{n} P_{o}}{\partial X_{a}^{n}} \\
& =\frac{(-1)^{n}}{\delta^{n}} K_{\mathrm{GS}} D F_{n}\left(P_{o}\right)
\end{aligned}
$$

where $F_{n}\left(P_{o}\right)=\sum_{k=1}^{n} D_{n, k} P_{o}^{k}$ is a polynomial in $P_{o}$ of order $n+1$ and with coefficients $D_{n, k}$. Given the Taylor expansions in eq. (10), the correction terms with no dependence on $\delta_{p}$ can therefore be written as

$$
\frac{1}{n !} \frac{\partial^{n} f_{X_{a}}}{\partial X_{a}^{n}}\left\langle\delta_{a, p}^{n}\right\rangle=\frac{(-1)^{n}}{n !} C_{1} F_{n}\left(P_{o}\right)\left\langle\left(\frac{\delta_{a, p}}{\delta}\right)^{n}\right\rangle
$$


and

$$
\frac{1}{n !} \frac{\partial^{n} f_{X}}{\partial X_{a}^{n}}\left\langle\delta_{a, p}^{n}\right\rangle=\frac{(-1)^{n}}{n !} C_{2} F_{n}\left(P_{o}\right)\left\langle\left(\frac{\delta_{a, p}}{\delta}\right)^{n}\right\rangle,
$$

respectively, where the average here is a population average over all hair bundles in the system, $C_{1}=\left(F_{\max } S-\right.$ $\left.K_{\mathrm{GS}} D\right)$, and $C_{2}=K_{\mathrm{GS}} D$. A numerical investigation suggests (not shown) that a constant $C$ exists, such that

$$
\left|F_{n}\left(P_{o}\right) / n !\right|<C
$$

for all $n$ and $0 \leq P_{o} \leq 1$. This implies that correction terms are expected not to have an impact on mean-field dynamics, when $\left|\delta_{a}\right| / \delta \ll 1$, i.e. loosely speaking, when the distribution of motors is more narrow than the effective range of the nonlinearity in the function $P_{o}$. As pointed out above, $T_{a}$ controls to what extend the ensemble of motors is smeared out compared to $\delta$. One can get a rough estimate up to what effective temperature the proposed mean-field theory can be used. To this end, in eq. (15) we neglect the contribution depending on the mean field (specifically, the term proportional to $P_{o}\left(\bar{X}, \bar{X}_{a}\right)\left[1-P_{o}\left(\bar{X}, \bar{X}_{a}\right)\right]$ which is in the oscillatory regime typically rather small) and also the effect of the second noise term. We thus consider the dynamic equation

$$
\lambda_{a} \dot{\delta}_{a}=-K_{\mathrm{GS}} \delta_{a}+\xi_{a}(t)
$$

where we have dropped the subscript $p$ for notational clarity. According to this equation, the variance of $\delta_{a}$ is given by

$$
\left\langle\delta_{a}^{2}\right\rangle=\frac{k_{B} T_{a}}{K_{\mathrm{GS}}} .
$$

Consequently, we expect the mean-field equations to be a good approximation, whenever

$$
T_{a} \ll \frac{\delta^{2} K_{\mathrm{GS}}}{k_{B}} \approx 1000 K
$$

For standard parameters, $T_{a}=450 \mathrm{~K}$, and thus $T_{a}$ differs from the right-hand side of the above inequality only by a factor of two. Therefore, we can only expect limited quantitative agreement of our theory with simulations of systems of coupled hair bundles.

\section{Comparison to the dynamics of coupled hair bundles}

In this section, we compare the spontaneous and driven dynamics of the mean field of systems of strongly coupled hair bundles (referred to as "coupled hair bundles (CHBs)"), the dynamics of the stochastic mean field governed by eq. (22) (referred to as "stochastic mean field (SMF)"), and the dynamics of a single hair bundle with reduced noise (referred to as "bundle with reduced noise (BRN)"). In particular, we show that for all system sizes $N^{2}$, the SMF captures the dynamical effects of coupling more faithfully than the BRN. (a)

(b)
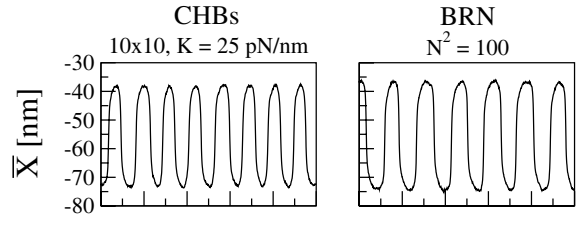

(c)
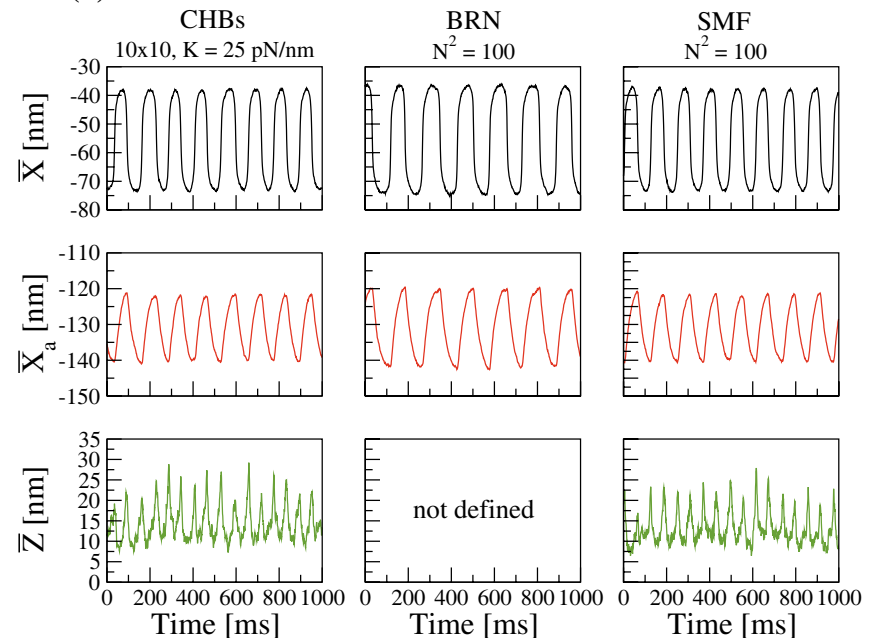

Fig. 4. Comparison to simulations of coupled hair bundles. Trajectories of the mean-field variables, $\bar{X}$ and $\bar{X}_{a}$, and, where applicable, of the population variance of motor deviations, $\bar{Z}$, are shown in (a) for a system of $10 \times 10$ coupled hair bundles $(\mathrm{CHBs})$ in the strong coupling regime $(K=25 \mathrm{pN} / \mathrm{nm})$, in (b) for a single hair bundle with reduced noise (BRN, $\varepsilon=1 / 100)$, and in (c) for the stochastic mean-field description (SMF, $N^{2}=$ 100).

\subsection{Spontaneous activity}

We commence with an investigation of the spontaneous activity. In fig. 4 , time traces of the mean-field variables, $\bar{X}$ and $\bar{X}_{a}$, are shown for $10 \times 10$ CHBs in the strong-coupling regime $(K=25 \mathrm{pN} / \mathrm{nm})$, the $\mathrm{BRN}$ with $\varepsilon=1 / 100$, and the SMF with $N^{2}=100$. In case of the CHBs and the SMF, also the time courses of the variable $\bar{Z}$ are displayed. All three oscillators exhibit spontaneous oscillations of their respective deflection variables, which are of higher regularity than for a single hair bundle (see fig. 2a, upper panel). However, oscillations of the BRN are of larger amplitude and lower frequency as compared to both the CHBs and the SMF. The dynamics of the latter qualitatively agree, including the behavior of the new variable $\bar{Z}$. In both cases, spikes of comparable amplitude can be discerned in $\bar{Z}$ during switches of the deflections of the CHBs and the SMF, respectively.

We extend our comparison in terms of spectral characteristics in fig. 5. More specifically, in fig. 5a, power spectral densities corresponding to the mean field of $5 \times 5 \mathrm{CHBs}$ $(K=100 \mathrm{pN} / \mathrm{nm})$, the BRN $(\varepsilon=1 / 25)$, and the SMF $\left(N^{2}=25\right)$ are shown. In agreement with the statements above, while the characteristic frequencies of the CHBs and the SMF are almost identical, the frequency of the $\mathrm{BRN}$ is markedly lower. Moreover, note that the shapes of the spectra of CHBs and SMF are in good agreement.

In sect. 3.2 , we have argued that for finite $T_{a}$, the dynamics given in eq. (22) are expected to only approximate the mean-field dynamics of the CHBs. According to our estimate, this approximation is expected to give reasonable results for $T_{a} \ll 1000 \mathrm{~K}$. In fig. $5 \mathrm{~b}$, we plot the char- 
(a)

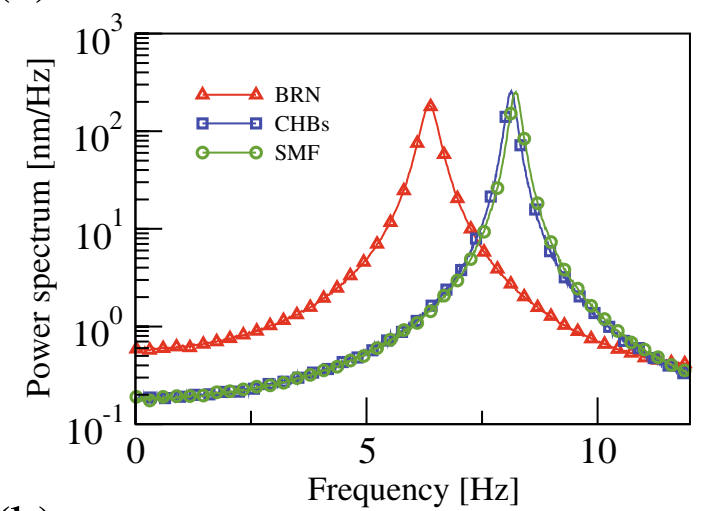

(b)

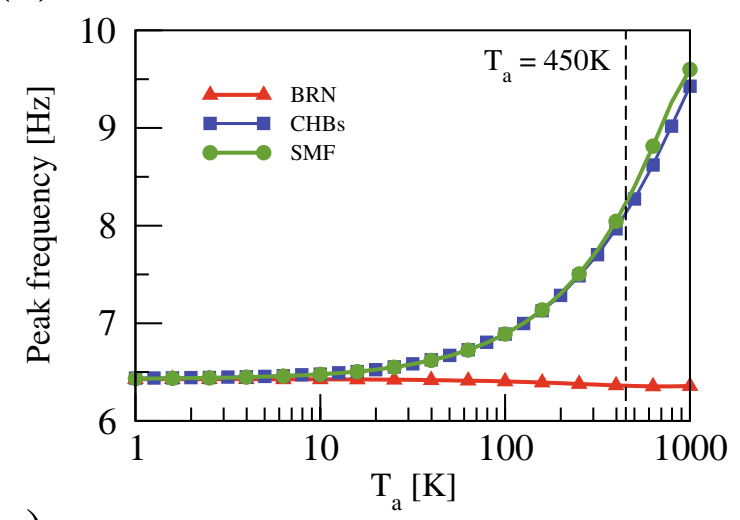

(c)

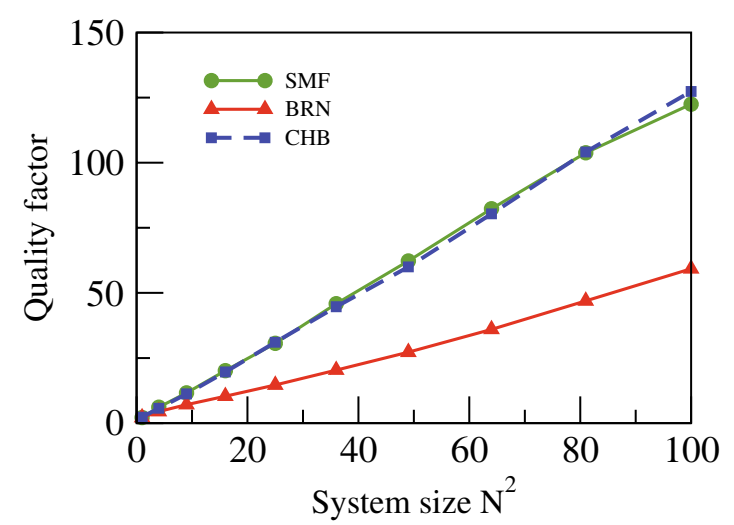

Fig. 5. Power spectra, frequency, and quality of coupled HBs (CHBs), stochastic mean field (SMF), and single HB with reduced noise (BRN), respectively. (a) Power spectra of the deflection variables, showing a pronounced difference in peak frequency for the coupled system and the single HB with reduced noise. (b) Peak frequencies of power spectra as functions of the effective temperature $T_{a}$. The spectra in panel (a) correspond to $T_{a}=450 \mathrm{~K}$. (c) Quality factors of the first peak in the spectra as functions of the system size.

acteristic frequency of the first spectral peak for CHBs, BRN, and SMF, respectively, as a function of $T_{a}$. While the frequency of the BRN with increasing effective temperate $T_{a}$ is slightly decreasing, for both CHBs and SMF an increase can be discerned. For all $T_{a}$ shown, the SMF gives a good approximation to the results for the CHBs.
However, for effective temperatures $T_{a}>400 \mathrm{~K}$, small deviations start to appear, indicative of the approximate nature of our mean-field approach. Hence, in particular for standard parameters, for which $T_{a}=450 \mathrm{~K}$ (see dashed vertical line fig. 5b), our approach gives reasonable results, however with minor deviations from the mean-field dynamics of CHBs.

Coupling-induced enhancement of the quality factor of spontaneous oscillations has been discussed as one of the important effects of elastic coupling [30]. In fig. 5, we show that, indeed, the quality factor of CHBs, BRN, and $\mathrm{SMF}$ increases in a linear fashion with system size. Here, we consider a noise reduction in the BRN with $\varepsilon=1 / N^{2}$. However, the results of the BRN underestimate the quality of CHBs and SMF. The latter quantitatively agree, again attesting that our approximate equations eq. (22) give reasonable results in capturing the effect of motor deviations on mean-field dynamics.

\subsection{Sensitivity with respect to periodic forcing}

In order to investigate whether our approximate meanfield approach also captures the enhanced sensitivity of systems of elastically coupled hair bundles, in this section, we study the response to periodic driving. In fig. 6a, we show the sensitivity to a sinusoidal driving (defined in sect. 2) as a function of driving amplitude, $F_{0}$, for CHBs $(5 \times 5, K=100 \mathrm{pN} / \mathrm{nm}), \mathrm{BRN}(\varepsilon=1 / 25)$, and $\mathrm{SMF}$ $\left(N^{2}=25\right)$. In all cases, the driving frequency, $\nu$, was chosen to coincide with the characteristic frequency, $f_{0}$, of the respective spontaneous activity. In all cases, three response regimes can be singled out: a high-sensitivity linear response regime for weak driving, a nonlinear decay of sensitivity for intermediate driving amplitudes, and a low-sensitivity linear response regime for strong driving. However, while all dynamics share these qualitative features, the CHBs and SMF quantitatively agree, with the BRN showing deviations. In particular, sensitivity in linear response to weak driving is higher for CHBs and SMF, respectively. As for the quality factor, for all three oscillators, a linear increase along with system size is found when the amplification gain (ratio of the two linear response regimes, defined in 2) is plotted as a function of system size (see fig. 6b). However, while the SMF captures the increase of the gain of the CHBs quantitatively, the BRN gives an underestimate.

\subsection{Effects of coupling beyond noise reduction}

As the results in the last two subsections show, the approximate mean-field approach eq. (22) quantitatively captures the mean-field dynamics of systems of elastically coupled hair bundles. In contrast, a single hair bundle with reduced noise exhibits appreciable deviations from both SMF and CHBs. This attests the fact that coupling has additional dynamic effects beyond simple noise reduction. These effects will be investigated in this subsection. 
(a)

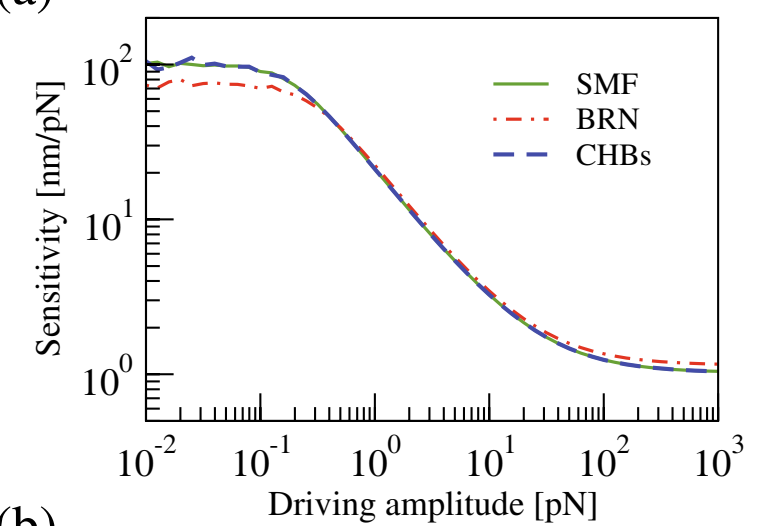

(b)

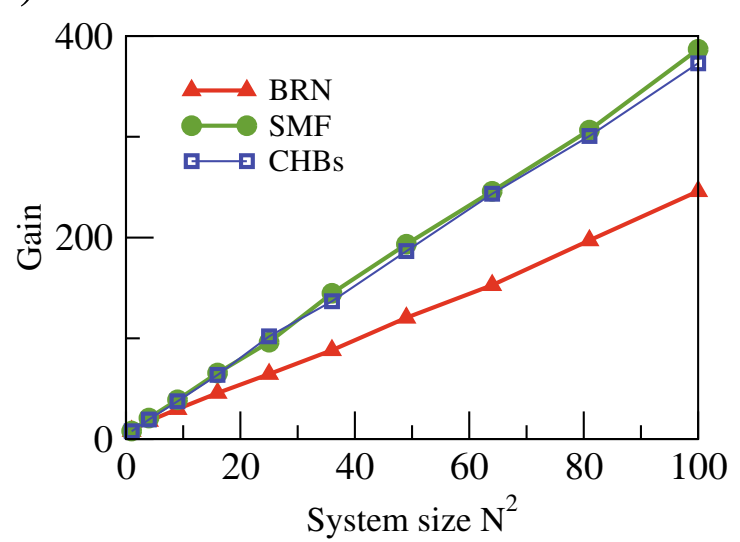

Fig. 6. Nonlinear response of the coupled system to a periodic stimulus at the best frequency. (a) Sensitivity vs. amplitude for the system of coupled HBs (CHBs), the stochastic mean field (SMF), and the single HB with reduced noise (BRN). (b) Gain as functions of system size.

To this end, we will first consider the infinite $N$ limit of the SMF. In this limit, noise terms in the dynamic eq. (22) vanish, rendering the SMF a deterministic dynamical system. In fig. 7a, the bifurcation diagram of the resulting system is shown in the $F_{\max }-S$-plane (orange lines). As a comparison, also the bifurcation diagram of a single deterministic hair bundle is displayed (black lines) [23], corresponding to a choice $\varepsilon=0$ in eq. (2). Note, the resulting system can also be viewed as the BRN in the limit of infinite $N$. Both bifurcation diagrams were obtained numerically with the help of the software package MATCONT [34], which allows for parameter continuation of equilibrium states and periodic orbits of autonomous systems of ODEs. Both systems show analogous regions in their bifurcation diagram. First of all, regions with one stable fixed point exist. Depending on the open probability of ion channels in this stationary state, in fig. 7a these regions are marked MO (mostly open) and MC (mostly closed). Secondly, a region of bistable behavior (marked BI in fig. 7a) can be discerned. Finally, within a bounded region of parameter space, both systems perform limit cycle oscillations (marked OSC in fig. 7a). Along the boundary (a)
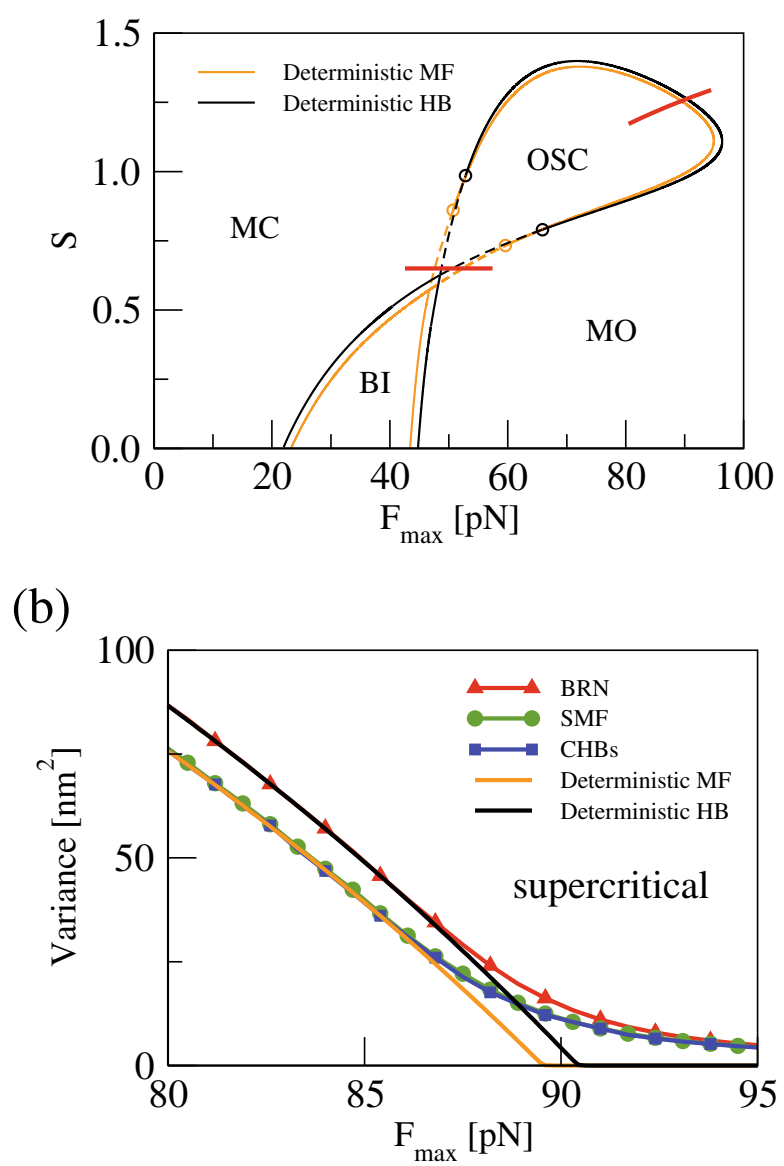

(c)

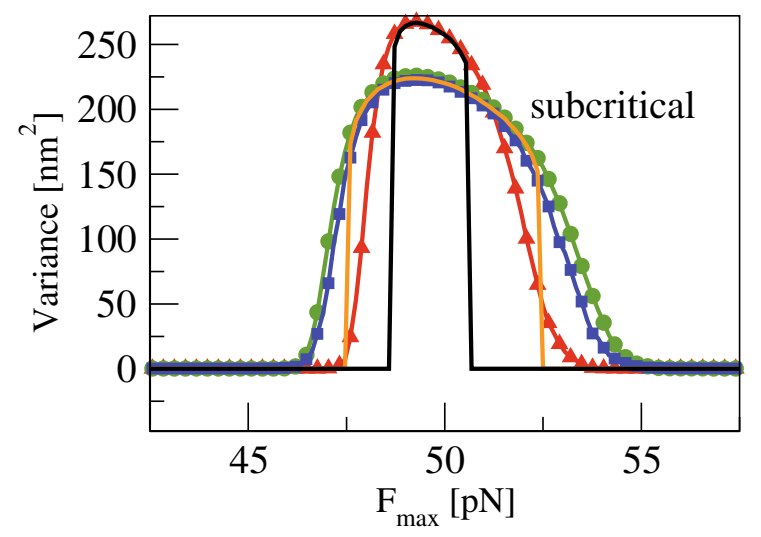

Fig. 7. (Colour on-line) Coupling-induced changes of the bifurcation structure. (a) Bifurcation diagram of the single HB and the mean-field theory in the limit of infinite $N$. A region of oscillatory dynamics (OSC) is enclosed by Hopf bifurcation lines (supercritical; solid lines; subcritical: dashed lines). (b) Variance when passing through the supercritical Hopf bifurcation for coupled HBs (CHBs), the stochastic mean field (SMF), single $\mathrm{HB}$ with reduced noise (BRN), deterministic hair bundle, and deterministic mean field; path corresponds to the upper red line in panel (a). (c) Variance when passing through the subcritical bifurcation along the lower red line in panel (a). 
of this oscillating region Hopf bifurcations occur (supercritical, solid lines; subcritical, dashed lines). While the qualitative features of the bifurcation diagrams are analogous, slight quantitative shifts of the bifurcation lines are apparent. For one, in the vicinity of the supercritical Hopf bifurcation, the oscillating region of the BRN extends to larger values of $F_{\max }$ and $S$ as compared to the SMF. In other words, there exists a small region in which the single deterministic hair bundle is oscillating, the SMF, however, is not. Coupling in this parameter regime is thus expected to suppress oscillations. In contrast, close to the subcritical bifurcation, the oscillating region of the BRN is more narrow than compared to the SMF. For parameter choices from between the respective bifurcation lines, systems of coupled hair bundles are expected to oscillate, even though in the absence of coupling, the individual hair bundles the system comprises are quiescent. We would like to emphasize that these changes in the deterministic bifurcation structure are caused by the finite fluctuations in the motor variable, i.e. the changes are noise-induced. This is somewhat similar to the noise-induced oscillations theoretically predicted by means of a cumulant expansion in [35]. The main differences to our system are that in [35] i) the multiplicative nature of the noise is essential for the change in the bifurcation structure of the mean field; ii) the noise acts only in the dynamics of the coupled variable, i.e. there is no finite variability in the uncoupled variable in the strong-coupling limit as it is found in our system.

In order to show that our results for the infinite $N$ limit also bear significance for finite system sizes, in fig. $7 \mathrm{~b}$ and c, we plot the variances of the deflection variables of the CHBs $(5 \times 5, K=100 \mathrm{pN} / \mathrm{nm}), \operatorname{SMF}\left(N^{2}=25\right)$, and BRN $(\varepsilon=1 / 25)$ along two lines in the $\left(F_{\max }, S\right)$-plane. While one is traversing the supercritical Hopf bifurcation, the other is intersecting the oscillating region via subcritical Hopf bifurcations (both paths are indicated as red lines in fig. 7a). We compare the results of stochastic simulations with the results of the deterministic systems (deterministic HB, deterministic MF). Close to the supercritical bifurcation, variances of CHBs and SMF coincide within line width. Furthermore, far from the bifurcation, where the variance is dominated by movements along the limit-cycle, both curves agree with the result for the deterministic mean field. Close to the bifurcation, where the amplitude of limit-cycle oscillations in the deterministic case go to zero, fluctuations start to dominate, effectively smearing out the bifurcation. However, also in this region CHBs and SMF give similar results. As far as the BRN is concerned, far from the bifurcation, it agrees with the results for a deterministic $\mathrm{HB}$, and deviates from the deterministic case close to the bifurcation. We conclude, that all three stochastic systems in the limit of large $N$, will approximate the curves for the respective deterministic systems. In particular, hallmarks of the different bifurcation structures of the two deterministic systems can also be appreciated at finite $N$. Similar observations can be made close to the subcritical bifurcation (see fig. 7c). Here, however, minor deviations of the SMF with respect to the CHBs emerge. (a)
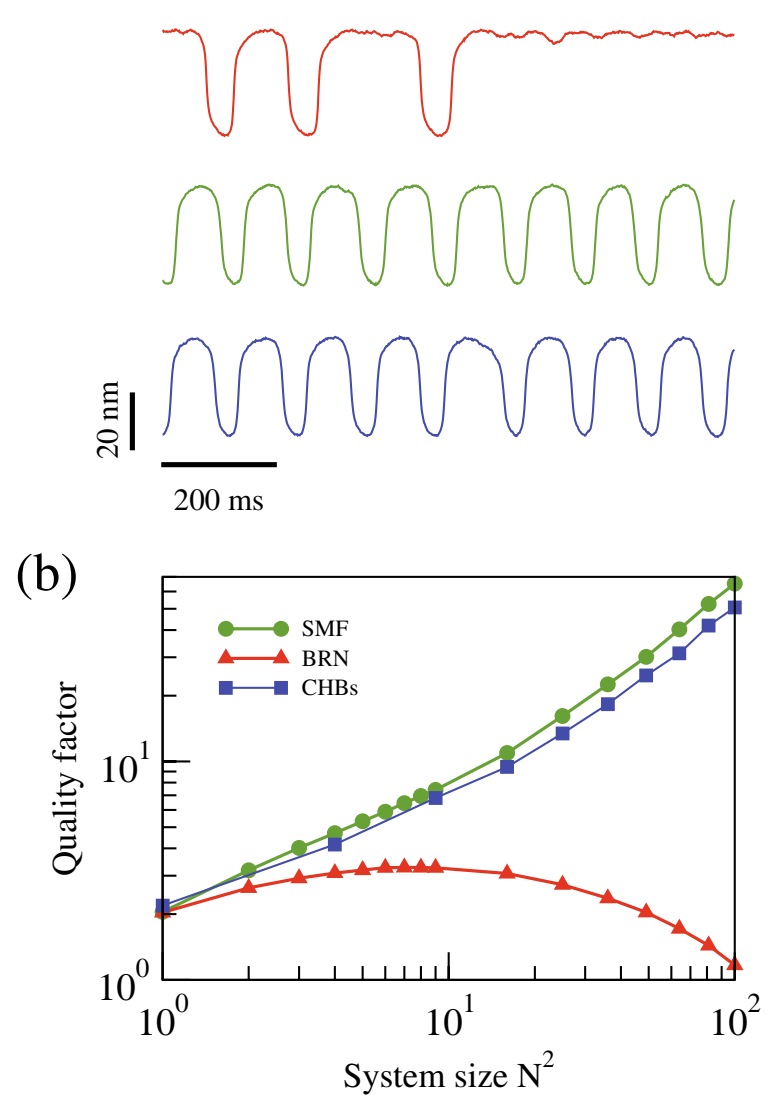

Fig. 8. (Colour on-line) Dynamics in the vicinity of the subcritical Hopf bifurcation. (a) Trajectories of a single hair bundle with reduced noise (red, $\varepsilon=1 / 100$ ), stochastic mean field (green, $N^{2}=100$ ), and coupled hair bundles (blue, $10 \times 10$ CHBs, $K=100 \mathrm{pN} / \mathrm{nm}$ ). (b) Quality as a function of $N$ near the subcritical bifurcation. Parameters, $S=0.65$ and $F_{\max }=51.1 \mathrm{pN} / \mathrm{nm}$, are chosen such that the single hair bundle resides outside the oscillatory regime, whereas the mean field for $N \rightarrow \infty$ is oscillating.

In the remainder of this subsection, we show that the observed changes in the bifurcation structure of the deterministic limits of BRN and SMF, can have pronounced effects when the statistics of the stochastic dynamics at finite $N$ are considered. To this end, we have chosen an operation point close to the subcritical bifurcation $\left(F_{\max }=51.1 \mathrm{pN} / \mathrm{nm}, S=0.65\right)$. More precisely, for this choice of parameters, while the deterministic mean field oscillates, the deterministic hair bundle is quiescent. Simulations of CHBs $(10 \times 10, K=100 \mathrm{pN} / \mathrm{nm})$ and $\mathrm{SMF}$ $\left(N^{2}=100\right)$ consequently result in noisy limit-cycle oscillations (see fig. 8a, color code in b), whose quality increases linearly along with system size (see fig. $8 \mathrm{~b}$ ). The BRN ( $\varepsilon=1 / 100)$, however, being close to a subcritical Hopf bifurcation, resides within an excitable regime, resulting in large noise-induced excursions along the precursor of a limit-cycle (see fig. 8a, red). The corresponding quality factor, when displayed as a function of system size, 
i.e. $\varepsilon=1 / N^{2}$, goes through a shallow maximum, indicative of coherence resonance $[36,8]$. At about $N^{2}=100$ it drops below one, i.e. coherent oscillations are lost. In other words, close to the subcritical Hopf bifurcation, elastic coupling of hair bundles can lead to an increase of regularity of oscillations. However, this enhancement does not have its origin in noise-reduction alone, but also in coupling-induced dynamic effects changing the overall dynamics of the system.

\section{Discussion}

On the basis of an existing stochastic biophysical description, we have developed in this paper a mean-field approach to the dynamics of systems of strongly coupled hair bundles. From a dynamic systems point of view, single hair bundle dynamics is described by a two-dimensional limit-cycle oscillator. Coupling only acts on one mechanical degree of freedom, i.e. the deflection variable. We have derived dynamic equations, capturing the stochastic dynamics of the mean field of such systems of strongly coupled hair bundles. The arguments presented shed light onto the dynamic origin of the effective noise reduction in systems of coupled hair bundles reported earlier. In particular, we have shown that in order to approximate mean-field dynamics faithfully, a new dynamic variable, namely the population variance of motor deviations from the mean field, needs to be taken into account. We have shown in numerical simulations, that this approach for all system sizes quantitatively describes the mean-field dynamics of systems of coupled hair bundles and also captures dynamic effects beyond mere noise reduction. We have argued, that e.g. close to the subcritical Hopf bifurcation of the deterministic hair-bundle system, qualitative differences can appear between the dynamics of systems of coupled hair bundles and a single hair bundle with reduced noise. These discrepancies are also reflected in the dynamics of the stochastic mean field as presented here.

Here, we have used a specific biophysical description of hair bundles that was shown to capture the essential characteristics observed for hair bundles of the sacculus of the bullfrog and the rat cochlea [31]. Other models for active hair bundle motility have been proposed. It has been suggested that calcium-mediated reclosure of mechanotransduction channels plays an important role for active hair bundle movements $[37,38]$.

Motivated by measurements of the material properties of the tectorial membrane, we have focused here on a purely elastic coupling among hair bundles. Other studies, concerned with the inner ear of the lizard [39] and gecko [40], have considered coupling of hair bundles to also have a viscous component.

Irrespective of the biophysical details, we expect hairbundle oscillations to synchronize when the deflection variables are effectively clamped by a strong coupling force, giving rise to an oscillatory mean field. Fluctuations in the uncoupled variables are expected in general to lead to modifications in the mean-field dynamics as compared to the isolated hair bundle and can give rise to additional degrees of freedom. The main effects of a strong coupling of displacement variables, discussed in this paper, should therefore also be relevant in other models of coupled hair bundles.

We would like to thank Pascal Martin and Jérémie Barral for stimulating discussions on hair bundle coupling. This work has been partly supported by the BMBF (grant FKZ: 01GQ1001A).

\section{Appendix A. Cross-correlation of noise terms in the stochastic mean-field dynamics}

First we state, that

$$
\left\langle\bar{\xi}(t) \bar{\xi}_{a}\left(t^{\prime}\right)\right\rangle \equiv 0
$$

for all $t, t^{\prime}$, because $\bar{\xi}(t)$ and $\bar{\xi}_{a}(t)$ are sums of distinct sets of mutually independent noise sources $\xi_{p}(t)$ and $\xi_{a, p}(t)$, respectively.

Next, we argue that the correlations between $\eta(t)$ and $\bar{\xi}_{a}(t)$ can be neglected (an analogous argument holds for the correlation between $\bar{\xi}(t)$ and $\eta(t))$. The crosscorrelation can be written and transformed as follows (using the formal solution eq. (16))

$$
\begin{aligned}
& \left\langle\eta(t) \frac{1}{N^{2}} \sum_{q=1}^{N^{2}} \xi_{a, q}(t+\tau)\right\rangle=\left\langle\frac{1}{N^{2}} \sum_{p=1}^{N^{2}} \delta_{a, p} \xi_{a, p}(t) \frac{1}{N^{2}}\right. \\
& \left.\times \sum_{q=1}^{N^{2}} \xi_{a, q}(t+\tau)\right\rangle \\
& =\frac{1}{N^{4}} \sum_{q=1}^{N^{2}} \sum_{p=1}^{N^{2}}\left\langle\frac { 1 } { \lambda _ { a } } e ^ { \Phi ( t ) } \int _ { - \infty } ^ { t } \mathrm { d } s \left(\xi_{a, p}(s)\right.\right. \\
& \left.\left.-\frac{1}{N^{2}} \sum_{r=1}^{N^{2}} \xi_{a, r}(s)\right) e^{-\Phi(s)} \xi_{a, p}(t) \xi_{a, q}(t+\tau)\right\rangle \\
& \approx \frac{1}{N^{4}} \sum_{q=1}^{N^{2}} \sum_{p=1}^{N^{2}} \frac{1}{\lambda_{a}} e^{\Phi(t)} \int_{-\infty}^{t} \mathrm{~d} s\left(\left\langle\xi_{a, p}(s) \xi_{a, p}(t) \xi_{a, q}(t+\tau)\right\rangle\right. \\
& \left.-\frac{1}{N^{2}} \sum_{r=1}^{N^{2}}\left\langle\xi_{a, r}(s) \xi_{a, p}(t) \xi_{a, q}(t+\tau)\right\rangle\right) e^{-\Phi(s)} \quad(\mathrm{A} .1) \\
& =0,
\end{aligned}
$$

because the $\xi_{a, p}(t)$ are pairwise independent, deltacorrelated, and third moments of Gaussian variables vanish. The step from the second to the third line is an approximation. For simplicity, here we treat $\Phi(t)$ as if it were a deterministic external function. This is justified because the time-scales relevant for the cross- and autocorrelations discussed in this appendix are short. A similar argument will be used below. 


\section{Appendix B. Mean value and auto-correlation of the effective noise $\eta(t)$}

The average of the driving noise, $\eta(t)$, in the dynamics of $\bar{Z}(t)$ does not vanish as can be shown using again the formal solution eq. (16)

$$
\begin{aligned}
\langle\eta(t)\rangle= & \frac{1}{N^{2}} \sum_{p=1}^{N^{2}}\left\langle\xi_{a, p}(t) \delta_{a, p}(t)\right\rangle \\
\approx & \frac{1}{N^{2}} \sum_{p=1}^{N^{2}} \frac{1}{\lambda_{a}} e^{\Phi(t)} \int_{-\infty}^{t} \mathrm{~d} s\left\langle\left(\xi_{a, p}(s)\right.\right. \\
& \left.\left.-\frac{1}{N^{2}} \sum_{q=1}^{N^{2}} \xi_{a, q}(s)\right) \xi_{a, p}(t)\right\rangle e^{-\Phi(s)} \\
= & \frac{1}{N^{2}} \sum_{p=1}^{N^{2}} \frac{1}{\lambda_{a}} e^{\Phi(t)} \int_{-\infty}^{t} \mathrm{~d} s 2 \lambda_{a} k_{B} T_{a} \delta(s-t) \\
& \times\left(1-\frac{1}{N^{2}}\right) e^{-\Phi(s)} \\
= & \left(1-\frac{1}{N^{2}}\right) k_{B} T_{a} .
\end{aligned}
$$

Next, we calculate the auto-correlation function of $\eta$. For clarity, in this calculation we drop the subscript $a$,

$$
\begin{aligned}
\langle\eta(t) \eta( & t+\tau)\rangle=\left\langle\frac{1}{N^{2}} \sum_{p=1}^{N^{2}} \delta_{p}(t) \xi_{p}(t) \frac{1}{N^{2}}\right. \\
& \left.\times \sum_{q=1}^{N^{2}} \delta_{q}(t+\tau) \xi_{q}(t+\tau)\right\rangle \\
= & \frac{1}{N^{4}} \sum_{p} \sum_{q}\left\langle\delta_{p}(t) \xi_{p}(t) \delta_{q}(t+\tau) \xi_{q}(t+\tau)\right\rangle \\
= & \frac{1}{N^{4}} \sum_{p} \sum_{q}\left\langle\frac { 1 } { \lambda _ { a } } e ^ { \Phi ( t ) } \int _ { - \infty } ^ { t } \mathrm { d } s _ { 1 } \left(\xi_{p}\left(s_{1}\right)\right.\right. \\
& \left.-\frac{1}{N^{2}} \sum_{r=1}^{N^{2}} \xi_{r}\left(s_{1}\right)\right) e^{-\Phi(s)} \xi_{p}(t) \\
& \cdot \frac{1}{\lambda_{a}} e^{\Phi(t+\tau)} \int_{-\infty}^{t+\tau} \mathrm{d} s_{2}\left(\xi_{q}\left(s_{2}\right)\right. \\
& \left.\left.-\frac{1}{N^{2}} \sum_{l=1}^{N^{2}} \xi_{l}\left(s_{2}\right)\right) e^{-\Phi\left(s_{2}\right)} \xi_{q}(t+\tau)\right\rangle \\
\approx & \frac{1}{N^{4} \lambda_{a}^{2}} e^{\phi(t)+\phi(t+\tau)} \int_{-\infty}^{t} \int_{-\infty}^{t+\tau} \mathrm{d} s_{1} \mathrm{~d} s_{2} \\
& \times \Omega\left(t, t+\tau, s_{1}, s_{2}\right) e^{-\phi\left(s_{1}\right)} e^{-\phi\left(s_{2}\right)},
\end{aligned}
$$

where

$$
\begin{aligned}
& \Omega\left(t, t+\tau, s_{1}, s_{2}\right)=\sum_{p} \sum_{q}\left(\left\langle\xi_{p}\left(s_{1}\right) \xi_{q}\left(s_{2}\right) \xi_{p}(t) \xi_{q}(t+\tau)\right\rangle\right. \\
& -\frac{1}{N^{2}} \sum_{r}\left\langle\xi_{r}\left(s_{1}\right) \xi_{q}\left(s_{2}\right) \xi_{p}(t) \xi_{q}(t+\tau)\right\rangle \\
& -\frac{1}{N^{2}} \sum_{l}\left\langle\xi_{p}\left(s_{1}\right) \xi_{l}\left(s_{2}\right) \xi_{p}(t) \xi_{q}(t+\tau)\right\rangle \\
& \left.+\frac{1}{N^{4}} \sum_{r} \sum_{l}\left\langle\xi_{r}\left(s_{1}\right) \xi_{l}\left(s_{2}\right) \xi_{p}(t) \xi_{q}(t+\tau)\right\rangle\right) .
\end{aligned}
$$

We deal with the four summands in turn and use the fact that because $\xi_{p}(t)$ is Gaussian, one has

$$
\begin{aligned}
& \left\langle\xi_{p}\left(t_{1}\right) \xi_{q}\left(t_{2}\right) \xi_{r}\left(t_{3}\right) \xi_{l}\left(t_{4}\right)\right\rangle=\left\langle\xi_{p}\left(t_{1}\right) \xi_{q}\left(t_{2}\right)\right\rangle\left\langle\xi_{r}\left(t_{3}\right) \xi_{l}\left(t_{4}\right)\right\rangle \\
& +\left\langle\xi_{p}\left(t_{1}\right) \xi_{r}\left(t_{3}\right)\right\rangle\left\langle\xi_{q}\left(t_{2}\right) \xi_{l}\left(t_{4}\right)\right\rangle+\left\langle\xi_{p}\left(t_{1}\right) \xi_{l}\left(t_{4}\right)\right\rangle\left\langle\xi_{q}\left(t_{2}\right) \xi_{r}\left(t_{3}\right)\right\rangle
\end{aligned}
$$

for all choices $p, q, r, l$ between 1 and $N^{2}$ (see e.g. [41]). Then the first term in eq. (B.3) gives

$$
\begin{aligned}
& \sum_{p, q}\left\langle\xi_{p}\left(s_{1}\right) \xi_{q}\left(s_{2}\right) \xi_{p}(t) \xi_{q}(t+\tau)\right\rangle \\
& =\left(2 \lambda_{a} k_{B} T_{a}\right)^{2}\left(N^{2} \delta\left(s_{1}-s_{2}\right) \delta(\tau)+N^{2} \delta\left(t+\tau-s_{1}\right) \delta\left(t-s_{1}\right)\right. \\
& \left.+N^{4} \delta\left(t-s_{1}\right) \delta\left(t+\tau-s_{2}\right)\right) .
\end{aligned}
$$

The second term in eq. (B.3) gives

$$
\begin{aligned}
& -\frac{1}{N^{2}} \sum_{p, q, l}\left\langle\xi_{r}\left(s_{1}\right) \xi_{q}\left(s_{2}\right) \xi_{p}(t) \xi_{q}(t+\tau)\right\rangle \\
& =-\left(2 \lambda_{a} k_{B} T_{a}\right)^{2}\left(\delta\left(s_{1}-s_{2}\right) \delta(\tau)+\delta\left(t+\tau-s_{1}\right) \delta\left(t-s_{1}\right)\right. \\
& \left.+N^{2} \delta\left(t-s_{1}\right) \delta\left(t+\tau-s_{2}\right)\right) .
\end{aligned}
$$

Similarly, the third term in eq. (B.3) gives

$$
\begin{aligned}
& -\frac{1}{N^{2}} \sum_{p, q, l}\left\langle\xi_{p}\left(s_{1}\right) \xi_{l}\left(s_{2}\right) \xi_{p}(t) \xi_{q}(t+\tau)\right\rangle \\
& =-\left(2 \lambda_{a} k_{B} T_{a}\right)^{2}\left(\delta\left(s_{1}-s_{2}\right) \delta(\tau)+\delta\left(t+\tau-s_{1}\right) \delta\left(t-s_{1}\right)\right. \\
& \left.+N^{2} \delta\left(t-s_{1}\right) \delta\left(t+\tau-s_{2}\right)\right) .
\end{aligned}
$$

Finally, the last term in eq. (B.3) gives

$$
\begin{aligned}
& \frac{1}{N^{4}} \sum_{p, q, r, l}\left\langle\xi_{r}\left(s_{1}\right) \xi_{l}\left(s_{2}\right) \xi_{p}(t) \xi_{q}(t+\tau)\right\rangle \\
& =\left(2 \lambda_{a} k_{B} T_{a}\right)^{2}\left(\delta\left(s_{1}-s_{2}\right) \delta(\tau)+\delta\left(t+\tau-s_{1}\right) \delta\left(t-s_{2}\right)\right. \\
& \left.+\delta\left(t-s_{1}\right) \delta\left(t+\tau-s_{2}\right)\right)
\end{aligned}
$$

One thus finds

$$
\begin{aligned}
& \Omega\left(t, t+\tau, s_{1}, s_{2}\right)=\left(2 \lambda_{a} k_{B} T_{a}\right)^{2}\left(\left(N^{2}-1\right)^{1} \delta\left(s_{1}-s_{2}\right) \delta(\tau)\right. \\
& +\left(N^{2}-1\right)^{1} \delta\left(t+\tau-s_{1}\right) \delta\left(t-s_{2}\right) \\
& \left.+\left(N^{2}-1\right)^{2} \delta\left(t-s_{1}\right) \delta\left(t+\tau-s_{2}\right)\right) .
\end{aligned}
$$


For the correlation function of $\eta$ this finally leads to

$$
\begin{aligned}
& \langle\eta(t) \eta(t+\tau)\rangle \approx \frac{\left(2 \lambda_{a} k_{B} T_{a}\right)^{2}}{N^{4} \lambda_{a}^{2}}\left(N^{2}-1\right) e^{\phi(t)+\phi(t+\tau)} \\
& \times \int_{-\infty}^{t} \int_{-\infty}^{t+\tau} \mathrm{d} s_{1} \mathrm{~d} s_{2} \delta\left(s_{1}-s_{2}\right) \delta(\tau) e^{-\phi\left(s_{1}\right)} e^{-\phi\left(s_{2}\right)} \\
& +\frac{\left(2 \lambda_{a} k_{B} T_{a}\right)^{2}}{N^{4} \lambda_{a}^{2}}\left(N^{2}-1\right) e^{\phi(t)+\phi(t+\tau)} \\
& \times \int_{-\infty}^{t} \int_{-\infty}^{t+\tau} \mathrm{d} s_{1} \mathrm{~d} s_{2} \delta\left(t+\tau-s_{1}\right) \delta\left(t-s_{2}\right) e^{-\phi\left(s_{1}\right)} e^{-\phi\left(s_{2}\right)} \\
& +\frac{\left(2 \lambda_{a} k_{B} T_{a}\right)^{2}}{N^{4} \lambda_{a}^{2}}\left(N^{2}-1\right)^{2} e^{\phi(t)+\phi(t+\tau)} \\
& \times \int_{-\infty}^{t} \int_{-\infty}^{t+\tau} \mathrm{d} s_{1} \mathrm{~d} s_{2} \delta\left(s_{1}-t\right) \delta\left(t+\tau-s_{2}\right) e^{-\phi\left(s_{1}\right)} e^{-\phi\left(s_{2}\right)} \\
& =\left(2 k_{B} T_{a}\right)^{2}\left(\frac{1}{N^{2}}-\frac{1}{N^{4}}\right) \delta(\tau) e^{2 \phi(t)} \int_{-\infty}^{t} \mathrm{~d} s_{1} e^{-2 \phi\left(s_{1}\right)} \\
& +\left(2 k_{B} T_{a}\right)^{2}\left(\frac{1}{N^{2}}-\frac{1}{N^{4}}\right) \cdot \delta_{0 \tau} \\
& +\left(1-\frac{1}{N^{2}}\right)^{2}\left(k_{B} T_{a}\right)^{2} .
\end{aligned}
$$

While the remaining integral is still dependent on time, and thus also the intensity of the noise acting on $\bar{Z}$, it can be viewed as the formal solution $D_{\bar{Z}}(t)$ of the deterministic equation

$$
\dot{D}_{\bar{Z}}=-\frac{2}{\lambda_{a}} a(t) D_{\bar{Z}}+1
$$

Open Access This is an open access article distributed under the terms of the Creative Commons Attribution License (http://creativecommons.org/licenses/by/3.0), which permits unrestricted use, distribution, and reproduction in any medium, provided the original work is properly cited.

\section{References}

1. S.M. Reppert, D.R. Weaver, Nature 418, 935 (2002).

2. D. Difrancesco, Annu. Rev. Physiol. 55, 455 (1993).

3. A.K. Engel, P. Fries, W. Singer, Nat. Rev. Neurosci. 2, 704 (2001).

4. B. Doiron, M.J. Chacron, L. Maler, A. Longtin, J. Bastian, Nature 421, 539 (2003).

5. G. Buzsáki, Rhythms of the brain (Oxford University Press, 2006).

6. A.J. Hudspeth, Neuron 59, 530 (2008).

7. A. Pikovsky, M. Rosenblum, J. Kurths, Synchronization: A Universal Concept in Nonlinear Sciences (Cambridge University Press, Cambridge, UK, 2001).

8. B. Lindner, J. Garcia-Ojalvo, A. Neiman, L. SchimanskyGeier, Phys. Rep. 392, 321 (2004).

9. K. Kruse, F. Jülicher, Curr. Opin. Cell Biol. 17, 20 (2005). 10. J.R. Clay, R.L. DeHaan, Biophys. J. 28, 377 (1979).
11. P. Martin, D. Bozovic, Y. Choe, A.J. Hudspeth, J. Neurosci. 23, 4533 (2003).

12. D. Bell-Pedersen, M. Cassone, D. Earnest, S. Golden, P. Hardin, T. Thomas, M. Zoran, Nat. Rev. Genet. 6, 544 (2005).

13. D. Freeman, K. Masaki, A. McAllister, J. Wei, T. Weiss, Hear. Res. 180, 11 (2003).

14. K. Horikawa, K. Ishimatsu, E. Yoshimoto, S. Kondo, H. Takeda, Nature 441, 719 (2006).

15. J. Barral, K. Dierkes, B. Lindner, F. Jülicher, P. Martin, Proc. Natl. Acad. Sci. U.S.A. 107, 8079 (2010).

16. J.A. Acebrón, L.L. Bonilla, C.J.P. Vicente, F. Ritort, R. Spigler, Rev. Mod. Phys. 77, 137 (2005).

17. A.T. Winfree, J. Theor. Biol. 16, 15 (1967).

18. Y. Kuramoto, Chemical Oscillations, Waves and Turbulence (Springer, New York, 1984).

19. N. Masuda, Y. Kawamura, H. Kori, New J. Phys. 12, 093007 (2010).

20. P. Martin, A.J. Hudspeth, F. Jülicher, Proc. Natl. Acad. Sci. U.S.A. 98, 14380 (2001).

21. P. Martin, A.J. Hudspeth, Proc. Natl. Acad. Sci. U.S.A. 96, 14306 (1999).

22. P. Martin, A.J. Hudspeth, Proc. Natl. Acad. Sci. U.S.A. 98, 14386 (2001).

23. B. Nadrowski, P. Martin, F. Jülicher, Proc. Natl. Acad. Sci. U.S.A. 101, 12195 (2004)

24. J.W. Gu, W. Hemmert, D.M. Freeman, A.J. Aranyosi, Biophys. J. 95, 2529 (2008).

25. R. Gueta, D. Barlam, R.Z. Shneck, I. Rousso, Proc. Natl. Acad. Sci. U.S.A. 103, 14790 (2006).

26. C.-P. Richter, G. Emadi, G. Getnick, A. Quesnel, P. Dallos, Biophys. J. 93, 2265 (2007).

27. A.V. Kondrachuk, Hear. Res. 143, 130 (2000).

28. C.E. Strimbu, D. Ramunno-Johnson, L. Fredrickson, K. Arisaka, D. Bozovic, Hear. Res. 256, 58 (2009).

29. G.A. Manley, C. Köppl, Hear. Res. 238, 3 (2008).

30. K. Dierkes, B. Lindner, F. Jülicher, Proc. Natl. Acad. Sci. U.S.A. 105, 18669 (2008).

31. J.-Y. Tinevez, F. Jülicher, P. Martin, Biophys. J. 93, 4053 (2007).

32. P. Martin, A.D. Mehta, A.J. Hudspeth, Proc. Natl. Acad. Sci. U.S.A. 97, 12026 (2000).

33. B. Lindner, K. Dierkes, F. Jülicher, Phys. Rev. Lett. 103, 250601 (2009).

34. A. Dhooge, W. Govaerts, Y.A. Kuznetsov, ACM Trans. Math. Softw. 29, 141 (2003).

35. R. Kawai, X. Sailer, L. Schimansky-Geier, C. Van den Broeck, Phys. Rev. E 69, 051104 (2004).

36. O.V. Ushakov, H.J. Wünsche, F. Henneberger, I.A. Khovanov, L. Schimansky-Geier, M.A. Zaks, Phys. Rev. Lett. 95, 123903 (2005).

37. Y. Choe, M.O. Magnasco, A.J. Hudspeth, Proc. Natl. Acad. Sci. U.S.A. 95, 15321 (1998).

38. A. Vilfan, T. Duke, Biophys. J. 85, 191 (2003).

39. A. Vilfan, T. Duke, Biophys. J. 95, 4622 (2008).

40. M. Gelfand, O. Piro, M. Magnasco, A.J. Hudspeth, PLoS ONE 5, e11116 (2010).

41. C.W. Gardiner, Handbook of Stochastic Methods (Springer-Verlag, Berlin, 2004). 